\title{
EXTENSIONS OF (SUPER) LIE ALGEBRAS
}

\author{
ALICE FIALOWSKI AND MICHAEL PENKAVA
}

\begin{abstract}
In this paper, we give a purely cohomological interpretation of the extension problem for (super) Lie algebras; that is the problem of extending a Lie algebra by another Lie algebra. We then give a similar interpretation of infinitesimal deformations of extensions. In particular, we consider infinitesimal deformations of representations of a Lie algebra.
\end{abstract}

\section{INTRODUCTION}

The notion of an extension of a (super) Lie algebra is an old idea in Lie theory (see 7, 8, 9]), even though not much explicit research has been done recently (see [1, 10, 11]). One of the difficulties may have been the lack of tools which help to identify and classify extensions of a given algebra. As we will point out in this paper, to give a satisfactory definition of the moduli space of all nonequivalent extensions of a Lie algebra, it is necessary to consider a more general notion of equivalence of extensions than usually appears in the literature.

The structure of this paper is as follows. After some preliminary definitions and explanation of notation in section 2, we will recall the classical definition of a super Lie algebra extension and discuss the notion of equivalent extensions in section 3. In section 4, we introduce a more general concept of equivalence of extensions, and give the classification of extensions of a Lie algebra in purely cohomological terms. To indicate the advantage of the general definition of equivalence, we give a few examples which could not be obtained with the restricted definition. In section [5, we define infinitesimal deformations of extensions of super Lie algebras, and in section [6 we introduce infinitesimal deformations of representations.

Our main guide in studying the extension problem comes from the recent studies of the moduli spaces of equivalence classes of Lie algebra structures on low dimensional spaces in [5, 6, ?]. The set of equivalence classes of extensions of a Lie algebra by another Lie algebra determines

Date: October 13, 2018.

The research of the authors was partially supported by OTKA grants T043641 and T043034 and by grants from the University of Wisconsin-Eau Claire. 
a moduli space as well, and the notion of equivalence we use is based on the philosophy that the moduli space of extensions should fit nicely into the moduli space of Lie algebra structures on the underlying vector space given by the sum of the two Lie algebras. A too restricted notion of equivalence leads to a moduli space which is too large.

\section{Preliminaries}

In classical Lie theory, the cohomology of a Lie algebra is studied by considering a differential on the space of maps from the exterior algebra of the vector space to the vector space itself; that is, the cochains with values in the adjoint representation. The exterior algebra $\bigwedge V$ of a non-graded vector space $V$ has a natural $\mathbb{Z}$-grading, which induces a $\mathbb{Z}_{2}$-grading on the exterior algebra. There is also a superbracket, the Richardson-Nijenhuis bracket [12], on this space of cochains, equipping it with the structure of a super Lie algebra. Thus, even for ordinary Lie algebras, one needs to introduce the notion of a $\mathbb{Z}_{2}$-graded space in order to understand deformation theory.

In this paper, we will find it is more convenient to consider Lie algebra structures as codifferentials in the space of coderivations of a symmetric coalgebra, rather than the exterior algebra, a language which is particularly well suited for generalization to other contexts, but which may not be familiar to Lie algebra theorists. Accordingly, we will provide here an explanation for our terminology. In fact, in the case of ordinary (not super) Lie algebras, only a dictionary need be provided, because the codifferentials we consider are simply Lie algebra structures, the coderivations we consider are simply elements of the cochain complex of the Lie algebra, and the $\mathbb{Z}_{2}$-grading of the coderivations is given only in terms of the degree of the cochain. The bracket of coderivations coincides with the usual Richardson-Nijenhuis bracket of cochains.

For super Lie algebras, it is also possible to study the cohomology without introducing the coderivation point of view, but the bracket of cochains is much simpler to express in terms of the bracket of coderivations; the signs which appear are simpler to express and easier to understand. The fact that the bracket structure of the cochains has a natural interpretation as a bracket of coderivations of a symmetric coalgebra was first noticed by J. Stasheff in [13].

Let $V$ be a $\mathbb{Z}_{2}$-graded vector space over a field $\mathbb{K}$, which, in our examples, we will assume is $\mathbb{C}$, although, for the most part, $\mathbb{K}$ can be any field whose characteristic is not 2 or 3 . Recall that a super Lie algebra structure on $V$ is given by a graded antisymmetric map 
$V \otimes V \rightarrow V$, denoted by $(a, b) \mapsto[a, b]$, which satisfies the graded Jacobi identity

$$
[a,[b, c]]=[[a, b], c]+(-1)^{a b}[b,[a, c]],
$$

for homogeneous elements. If $V=V_{e}$, then the structure is a Lie algebra, and the sign $(-1)^{a b}$ does not appear.

Recall that for a Lie algebra, the cochain complex with values in the adjoint representation, which is important for deformation theory, is given by $C(V)=\prod_{n=0}^{\infty} C^{n}(V)$, where $C^{n}(V)=\operatorname{Hom}\left(\bigwedge^{n} V, V\right)$. The space $C(V)$ has a $\mathbb{Z}_{2}$-grading, where the parity of an element in $C^{n}$ is even if $n$ is odd, and odd if $n$ is even. There is a bracket operation on $C(V)$, which satisfies $\left[C^{k}(V), C^{l}(V)\right] \subseteq C^{k+l-1}(V)$, and this bracket equips $C(V)$ with the structure of a $\mathbb{Z}_{2}$-graded Lie algebra. In fact, if $\varphi \in C^{k}(V), \psi \in C^{l}(V)$, and $n=k+l-1$ then we can define $\phi \circ \psi \in C^{n}(V)$ by

$$
\phi \circ \psi\left(v_{1}, \cdots, v_{n}\right)=\sum_{\sigma \in \operatorname{Sh}(l, k-1)}(-1)^{\sigma} \phi\left(\psi\left(v_{\sigma(1)}, \cdots, v_{\sigma(l)}\right), v_{\sigma(l+1)}, \cdots, v_{\sigma(n)}\right),
$$

where $(-1)^{\sigma}$ is the sign of the permutation $\sigma$ and $\operatorname{Sh}(l, k-1)$ are the permutations in $\Sigma_{n}$ which are increasing on the first $l$ and the last $k-1$ elements. The bracket of cochains is given by

$$
[\phi, \psi]=\phi \circ \psi-(-1)^{\phi \psi} \psi \circ \phi,
$$

where $(-1)^{\phi \psi}=(-1)^{(k-1)(l-1)}$.

The cohomology of the Lie algebra is determined by the differential $D: C(V) \rightarrow C(V)$, given by $D(\phi)=[l, \phi]$, where $l$ is the Lie algebra structure, interpreted as an element of $C^{2}(V)$. The fact that $D^{2}=$ 0 follows from the Jacobi identity, which in terms of cochains is the codifferential property $[l, l]=0$. Note that $l$ is an odd cochain, because it lies in $C^{2}(V)$. Therefore, by the graded Jacobi identity on $C(V)$, we have

$$
D^{2}(\phi)=[l,[l, \phi]]=[[l, l], \phi]-[l,[l, \phi]=-[l,[l, \phi]] .
$$

The formula above explains why we do not allow a field of characteristic 2 in our theory. The reason fields of characteristic 3 are a problem is more subtle, but has to do with the fact that we want the triple bracket $[\alpha,[\alpha, \alpha]]$ to vanish for any $\alpha$, and this will be true as long as the characteristic of the field is not 3. Even when the Lie algebra is not graded, the cochain complex $C(V)$ is a $\mathbb{Z}_{2}$-graded Lie algebra, so fields of characteristic 2 or 3 are still problematic.

When working with $\mathbb{Z}_{2}$-graded Lie algebras, it is convenient to consider the parity reversion $W=\Pi V$, where $W_{o}=V_{e}$ and $W_{e}=V_{o}$. We can identify $C(V)=\operatorname{Hom}(\bigwedge V, V)$ with $C(W)=\operatorname{Hom}(S(W), W)$, 
where $S(W)$ is the $\mathbb{Z}_{2}$-graded symmetric coalgebra of $W$. Note that the symmetric coalgebra coincides with the symmetric algebra as a vector space, equipped with the cocommutative, coassociative coproduct $\Delta: S(W) \rightarrow S(W) \otimes S(W)$, given by

$$
\Delta\left(w_{1} \cdots w_{n}\right)=\sum_{\substack{k=0 \ldots n \\ \sigma \in \operatorname{Sh}(k, n-k)}} \epsilon(\sigma) w_{1} \cdots w_{k} \otimes w_{k+1} \cdots w_{n},
$$

where $\epsilon(\sigma)$ is a sign determined by the rule

$$
w_{\sigma(1)} \cdots w_{\sigma(n)}=\epsilon(\sigma) w_{1} \cdots w_{n} .
$$

There is a natural identification of $C(W)$ with the coderivations of $S(W)$, and the bracket in $C(W)$ corresponds to the bracket of coderivations. In fact, if $\phi \in C^{k}(W), \psi \in C^{l}(W)$, and $n=k+l-1$, then we define $\phi \circ \psi \in C^{n}(W)$ by

$$
\phi \circ \psi\left(w 1 \cdots w_{n}\right)=\sum_{\sigma \in \operatorname{Sh}(l, k-1)} \epsilon(\sigma) \phi\left(\psi\left(w_{\sigma(1)} \cdots w_{\sigma(l)}\right) w_{\sigma(l+1)} \cdots w_{n}\right),
$$

Then

$$
[\phi, \psi]=\phi \circ \psi-(-1)^{\phi \psi} \psi \circ \phi
$$

where $(-1)^{\phi \psi}$ is a sign determined by the product of the parities of $\phi$ and $\psi$, rather than their degrees as cochains.

Note that when $V$ is an ordinary Lie algebra, $\epsilon(\sigma)=(-1)^{\sigma}$ is just the sign of the permutation, and the parity of the element in $C(W)$ associated to an element in $C^{k}(V)$ is just $k-1$, so the bracket in $C(W)$ is just the bracket in $C(V)$. Therefore, for ordinary Lie algebras, the construction of $C(W)$ is just a change of language.

If $d$ is the element in $C^{2}(W)$ associated to a Lie algebra structure $l$ in $C^{2}(V)$, then $d$ satisfies the following version of the Jacobi identity.

$$
d(d(a, b), c)+(-1)^{b c} d(d(a, c), b)+(-1)^{a(b+c)} d(d(b, c), a)=0 .
$$

In fact, $d$ is an odd coderivation of the symmetric coalgebra $S(W)$, and moreover the Jacobi identity above is simply the codifferential property $[d, d]=0$. We say that $d$ is an odd codifferential on $S(W)$.

One defines the cohomology of the codifferential $d$ on $W$ in terms of the differential $D: C(W) \rightarrow C(W)$ given by $D(\varphi)=[d, \varphi]$. We call the resulting homology the cohomology $H(d)$ of the super Lie algebra structure on $V$ given by the codifferential $d$ on $W$.

The main advantage in working with $C(W)$ instead of $C(V)$ is that in $C(W)$, one only has to consider the parity of an element, instead of its parity and its degree as a cochain. Thus we only have to consider the structure of $C(W)$ as a graded Lie algebra, which is a real advantage in computations. 
Let $\left\{w_{1}, \cdots, w_{n}\right\}$ be a basis of the space $W$, and let $w_{i, j}=w_{i} w_{j}$ denote the product of two basis elements, which lies in $S^{2}(W)$. Consider the map

$$
S(i):\{1, \cdots, n\} \rightarrow\{1, \cdots, n\} \times\{1, \cdots, n\}
$$

given by the sequence of ordered pairs

$$
(1,2),(1,3),(2,3),(1,4),(2,4), \cdots,(n-1, n) .
$$

Then there is a natural basis $\left\{w_{S(1)}, \cdots, w_{S\left(\begin{array}{c}n \\ 2\end{array}\right)}\right\}$ for $S^{2}(W)$, which we will use in order to represent codifferentials $d: S^{2}(W) \rightarrow W$ as matrices. Moreover, any element of $C^{2}(W)$ can be also represented as a sum $d=a_{i} \psi_{j}^{S(i)}$, where $\psi_{k}^{i, j}: S^{2}(W) \rightarrow W$ is defined by $\psi_{k}^{i, j}\left(w_{l, m}\right)=\delta_{l, m}^{i, j} w_{k}$, and we shall also express our codifferentials using this second notation in our examples.

\section{Extensions of Lie Algebras}

We consider the extension picture of a (super) Lie algebra $W$ by a Lie algebra $M$. In other words we consider the short exact sequence of Lie algebras

$$
0 \rightarrow M \rightarrow V \rightarrow W \rightarrow 0,
$$

For convenience, we introduce the following notation for certain spaces of cochains on $V=M \oplus W$.

$$
\begin{aligned}
C^{n} & =\operatorname{Hom}\left(W^{n}, W\right) \\
C^{k, l} & =\operatorname{Hom}\left(M^{k} W^{l}, M\right),
\end{aligned}
$$

where $M^{k} W^{l}$ is the subspace of $S^{k+l}(V)$ determined by products of $k$ elements from $M$ and $l$ elements from $W$. The Lie algebra structure on $V$ is determined by the following maps:

$$
\begin{array}{lr}
\delta \in C^{2}=\operatorname{Hom}\left(W^{2}, W\right): & \text { the Lie algebra structure on } W \\
\psi \in C^{0,2}=\operatorname{Hom}\left(W^{2}, M\right): & \text { the 2-cocycle on } M \\
\lambda \in C^{1,1}=\operatorname{Hom}(M W, M): & \text { the } W \text { - "module structure" on } M \\
\mu \in C^{2,0}=\operatorname{Hom}\left(M^{2}, M\right): & \text { the Lie algebra structure on } M
\end{array}
$$

The fact that $d$ has no terms from $\operatorname{Hom}\left(M W \oplus M^{2}, W\right)$ reflects the fact that $M$ is an ideal in $V$. The Jacobi identity is the condition that the coderivation $d=\delta+\lambda+\rho+\mu+\psi$ is a codifferential on $S(V)$, which means that $[d, d]=0$. Now, in general, we see that $[d, d] \in \operatorname{Hom}\left(S^{3}(V), V\right)$. 
By decomposing this space and considering which parts the brackets of the terms $\delta, \lambda \mu$ and $\psi$ are defined on, we obtain

$$
\begin{array}{ll}
\text { (2) } & {[\delta, \delta]=0: \text { The Jacobi identity for } \delta} \\
\text { (3) } & {[\mu, \mu]=0: \text { The Jacobi identity for } \mu \text {. }} \\
\text { (4) } & {[\delta, \lambda]+1 / 2[\lambda, \lambda]+[\mu, \psi]=0: \text { The "module" relation. }} \\
\text { (5) } & {[\mu, \lambda]=0: \text { The module-algebra structures are compatible. }} \\
\text { (6) } & {[\delta+\lambda, \psi]=0: \psi \text { is a 2-cocycle with values in } M .}
\end{array}
$$

We also have the automatic relations $[\mu, \delta]=[\psi, \psi]=0$. When $[\mu, \psi]=$ 0 , equation (44) says that $\lambda$ is a module structure in the usual sense, so this relation can be considered as saying that $\lambda$ determines a generalized module structure. Note that $\delta+\mu$ is a codifferential, the direct sum of the codifferentials on $M$ and $W$, and $\lambda+\psi$ satisfies the Maurer-Cartan formula (MC-formula)

$$
[\delta+\mu, \lambda+\psi]+\frac{1}{2}[\lambda+\psi, \lambda+\psi]=0,
$$

with respect to the codifferential $\delta+\mu$. Furthermore, $\mu+\lambda+\psi$ satisfies the MC formula

$$
[\delta, \mu+\lambda+\psi]+\frac{1}{2}[\mu+\lambda+\psi, \mu+\lambda+\psi]=0,
$$

with respect to the codifferential $\delta$. All of these basic facts about Lie algebra extensions are well known. (See, for example [7, 9].) We summarize the main facts in the theorem below.

Theorem 3.1. If $\delta$ is a Lie algebra structure on $W$ and $\mu$ is a Lie algebra structure on $M$, then $d=\delta+\mu+\lambda+\psi$, where $\lambda \in \operatorname{Hom}(M W, M)$ and $\psi \in \operatorname{Hom}\left(W^{2}, M\right)$ determines a Lie algebra structure on $M \oplus W$ precisely when the three conditions below hold:

$$
\begin{aligned}
& {[\delta, \lambda]+\frac{1}{2}[\lambda, \lambda]+[\mu, \psi]=0} \\
& {[\mu, \lambda]=0} \\
& {[\delta+\lambda, \psi]=0}
\end{aligned}
$$

\section{Equivalence of extensions of Lie Algebras}

A (restricted) equivalence of extensions of Lie algebras is given by a commutative diagram of the form

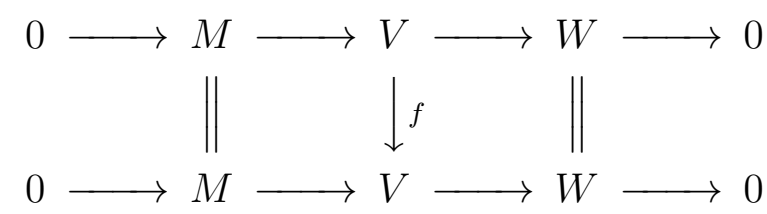


where we assume that in the top row, $V$ is equipped with the codifferential $d=\delta+\mu+\lambda+\psi$, and in the bottom row, it is equipped with the codifferential $d^{\prime}=\delta+\mu+\lambda^{\prime}+\psi^{\prime}$, and $f$ is a morphism of Lie algebras (which is necessarily an isomorphism). In order for the diagram to commute, we must have $f(m, w)=(m+\beta(w), w)$, where $\beta: W \rightarrow M$ is linear. Note that if $g(m, w)=(m-\beta(w), w)$, then $g=f^{-1}$.

The requirement that $f$ be a morphism of Lie algebras is simply that $d^{\prime}=g^{*} d=g^{-1} \circ d \circ g$, where $g$ is extended to an automorphism of $S(V)$. It follows that

$$
\begin{aligned}
\delta^{\prime} & =\delta \\
\mu^{\prime} & =\mu \\
\lambda^{\prime} & =\lambda-[\mu, \beta] \\
\psi^{\prime} & =\psi-\left[\delta+\lambda-\frac{1}{2}[\mu, \beta], \beta\right] .
\end{aligned}
$$

Since $d^{\prime}=g^{*} d$, it is automatically true that $d^{\prime}$ is a codifferential.

If we consider the map $\beta$ as an endomorphism of $V$, then $\beta^{2}=0$, and therefore $\exp (\beta)=1+\beta=f$. But then $f^{*}$ is given by the formula

$$
f^{*}=\exp \left(-\operatorname{ad}_{\beta}\right)
$$

which means that the action of $f^{*}$ on $\operatorname{Hom}(S(V), V)$ is expressible in terms of brackets. Note that

$$
\begin{array}{r}
\operatorname{ad}_{\beta}: C^{k} \rightarrow C^{0, k} \\
\operatorname{ad}_{\beta}: C^{k, l} \rightarrow C^{k-1, l+1} .
\end{array}
$$

The term $\frac{1}{2}[[\mu, \beta], \beta]$ is simply $\frac{1}{2} \operatorname{ad}_{\beta}^{2}(\mu)$, arising from the exponential, and this is the only nonvanishing term in $f^{*}(d)$ involving a double bracket with $\beta$, and all higher order brackets vanish.

An extension $V$ is said to be a semidirect product provided that $W$ is a subalgebra of $V$. This just means that $\psi=0$. Clearly, an extension is equivalent to a semidirect product precisely when there is some linear $\beta: W \rightarrow M$ such that $\psi=\left[\delta+\lambda-\frac{1}{2}[\mu, \beta], \beta\right]$. Let us summarize the results above in the following theorem, which is given in a slightly different form in [1].

Theorem 4.1. If $d=\delta+\mu+\lambda+\psi$ and $d^{\prime}=\delta+\mu+\lambda^{\prime}+\psi^{\prime}$ are two extensions of the Lie algebra structure $\delta$ on $W$ by a Lie algebra structure $\mu$ on $M$, then they are equivalent extensions precisely when 
there is a $\beta \in \operatorname{Hom}(W, M)$ such that

$$
\begin{aligned}
\lambda^{\prime} & =\lambda-[\mu, \beta] \\
\psi^{\prime} & =\psi-\left[\delta+\lambda-\frac{1}{2}[\mu, \beta], \beta\right] .
\end{aligned}
$$

Moreover the extension is equivalent to a semidirect product precisely when there is some $\beta \in \operatorname{Hom}(W, M)$ such that

$$
\psi=\left[\delta+\lambda-\frac{1}{2}[\mu, \beta], \beta\right] .
$$

The group $G_{\text {rest }}$ of automorphisms $f$ of $V$ of the form

$$
f(m, w)=(m+\beta(w), w)
$$

acts on the set of extensions. The set of equivalence classes of such extensions under this group action is the restricted moduli space of extensions of $\delta$ by $\mu$.

4.1. Infinitesimal extensions and infinitesimal equivalence. The notion of an infinitesimal extension is expressed in the form

$$
d=\delta+\mu+t(\lambda+\psi)
$$

where $t^{2}=0$ is an infinitesimal parameter. The conditions for $d$ to be an extension reduce to

$$
\begin{aligned}
& {[\delta, \lambda]+[\mu, \psi]=0} \\
& {[\mu, \lambda]=0} \\
& {[\delta, \psi]=0}
\end{aligned}
$$

If $\alpha \in C(V)$, then denote $D_{\alpha}=\operatorname{ad}_{\alpha}$. When $\alpha$ is odd and $[\alpha, \alpha]=0$, then $D_{\alpha}^{2}=0$, so $D_{\alpha}$ is called a coboundary operator on $C(V)$, and $H_{\alpha}=\operatorname{ker}\left(D_{\alpha}\right) / \operatorname{Im}\left(D_{\alpha}\right)$ is the cohomology induced by $\alpha$. An element $\phi$ such that $D_{\alpha}(\phi)=0$ is called a $D_{\alpha}$-cocycle, and its image $[\phi]_{\alpha}$ in $H_{\alpha}$ is a $D_{\alpha}$-cohomology class. An element of the form $D(\phi)$ is called a $D_{\mu}$-coboundary. The bracket on $C(V)$ descends to a bracket on $H_{\alpha}$, so $H_{\alpha}$ inherits the structure of a Lie superalgebra. Since $\delta, \mu$ and $\psi$ are all codifferentials, they determine coboundary operators.

Note that there is a certain symmetry in the roles of $\delta$ and $\mu$ in the above conditions for an infinitesimal extension, in the sense that if you interchange the codifferentials $\delta$ and $\mu$, and the cochains $\lambda$ and $\psi$, then the conditions are unchanged. We have

$$
\begin{aligned}
D_{\delta}: C^{k} \rightarrow C^{k+1} & D_{\delta}: C^{k, l} \rightarrow C^{k, l+1} \\
D_{\mu}: C^{k} \rightarrow 0 & D_{\mu}: C^{k, l} \rightarrow C^{k+1, l} .
\end{aligned}
$$


Since $[\delta, \mu]=0$, it follows that $D_{\delta}$ and $D_{\mu}$ anticommute. As a consequence,

$$
D_{\mu}: \operatorname{ker}\left(D_{\delta}\right) \rightarrow \operatorname{ker}\left(D_{\delta}\right),
$$

so we can define the cohomology $H_{\mu}(\operatorname{ker} \delta)$ determined by the restriction of $D_{\mu}$ to $\operatorname{ker}\left(D_{\delta}\right)$.

For simplicity, let us denote the cohomology class of a $D_{\mu}$-cocycle $\varphi$ by $\bar{\varphi}$. It is easily checked that $D_{\mu}$ and $D_{\delta+\lambda}$ anticommute, since $[\mu, \delta]=0$. It follows that

$$
D_{\mu}: \operatorname{ker}\left(D_{\delta}\right) \rightarrow \operatorname{ker}\left(D_{\delta}\right)
$$

which means that we can define the cohomology $H_{\mu}(\operatorname{ker}(\delta))$, determined by the restriction of $D_{\mu}$ to $\operatorname{ker}\left(D_{\delta}\right)$. The existence of a $\psi$ such that $[\delta, \lambda]+[\mu, \psi]=0$ and $[\delta, \psi]=0$ is equivalent to the assertion that $\overline{[\delta, \lambda]}=0$ in $H_{\mu}(\operatorname{ker}(\delta))$.

Note that even though the condition for the existence of a $\psi$ depends explicitly on $\lambda$, if the statement is true for $\lambda$, then it is valid for any element in $\bar{\lambda}$. This follows because if $\lambda$ is replaced by $\lambda^{\prime}=\lambda-[\mu, \beta]$ and $\psi$ by $\psi^{\prime}=\psi-[\delta+\lambda, \beta]$, where $\beta: W \rightarrow M$, then we obtain a new codifferential $d^{\prime}=\delta+\mu+t\left(\lambda^{\prime}+\psi^{\prime}\right)$, which is in fact infinitesimally equivalent to $d$. By infinitesimal equivalence, we mean an equivalence determined by an infinitesimal automorphism $f=\exp (t \beta)$, where $\beta: W \rightarrow M$. (Actually, this is a restricted version of infinitesimal equivalence. We will introduce a more general notion later.) Since $d^{\prime}=f^{*}(d)$, it follows that $\left[\delta, \lambda^{\prime}\right]+\left[\mu, \psi^{\prime}\right]=0$.

Now consider a fixed $\lambda$ such that $\overline{[\delta, \lambda]}=0$ in $H_{\mu}(\operatorname{ker}(\delta))$, and choose some $\psi$ such that $[\delta, \lambda]+[\mu, \psi]=0$. If $\psi^{\prime}=\psi+\tau$ is another solution, then $[\mu, \tau]=0$ and $[\delta, \tau]=0$. Now $[\mu, \delta]=0$,so $\bar{\delta}$ is defined in $H_{\mu}$. The cohomology $H_{\mu}$ inherits the structure of a graded Lie algebra, with

$$
[\bar{\alpha}, \bar{\beta}]=\overline{[\alpha, \beta]} \text {. }
$$

Since $[\bar{\delta}, \bar{\delta}]=\overline{[\delta, \delta]}=0, \bar{\delta}$ determines a coboundary operator $D_{\bar{\delta}}$ on $H_{\mu}$. Denote the cohomology of $D_{\bar{\delta}}$ by $H_{\mu, \delta}$, and the cohomology class of a $D_{\bar{\delta}}$-cocycle $\bar{\varphi}$ by $[\bar{\varphi}]$. Then $[\bar{\delta}, \bar{\tau}]=0$, so $\tau$ determines a cohomology class $[\bar{\tau}]$. On the other hand, suppose that $\bar{\tau}$ is any $D_{\bar{\delta}}$-cocycle. Then $[\bar{\delta}, \bar{\tau}]=0$ implies that $[\delta, \tau]$ is a $D_{\mu}$-coboundary. Since $[\delta, \tau] \in C^{0,3}$, this forces $[\delta, \tau]=0$. Thus, every $D_{\bar{\delta}}$-cocycle determines an extension.

We need to determine when an extension given by a $D_{\bar{\delta}}$-cocycle $\bar{\tau}$ is equivalent to the extension given by $\lambda$ and $\psi$. Suppose that $\bar{\tau}=$ $-D_{\bar{\delta}}(\bar{\beta})$. This is equivalent to the conditions $[\mu, \beta]=0$ and $\tau=-[\delta, \beta]$, which is equivalent to the condition $f^{*}(d)=d^{\prime}$, where $f=\exp (t \beta)$. 
Thus, infinitesimal equivalence classes are parameterized by $H_{\mu, \delta}$. We summarize these results in the following theorem.

Theorem 4.2. The infinitesimal extensions of the Lie algebra structure $\delta$ on $W$ by a Lie algebra structure $\mu$ on $M$ are completely classified by the set of $D_{\mu}$-cohomology classes $\bar{\lambda}$ arising from $\lambda \in \operatorname{Hom}(M W, M)$ which satisfy the formula

$$
\overline{[\delta, \lambda]}=0 \in H_{\mu}\left(\operatorname{ker}\left(D_{\delta+\lambda}\right)\right)
$$

together with the $D_{\bar{\delta}}$-cohomology classes $[\bar{\tau}]$ of $D_{\bar{\delta}}$-cocycles $\bar{\tau}$ arising from of $D_{\mu}$-cocycles $\tau \in \operatorname{Hom}\left(W^{2}, M\right)$.

\subsection{Classification of extensions of a Lie algebra by a module.}

We consider the special case when the structure $\lambda$ determines an actual module structure on $W$; in other words, when the MC formula

$$
[\delta, \lambda]+\frac{1}{2}[\lambda, \lambda]=0
$$

holds. In this case it follows that $[\mu, \psi]=0$, so that $\bar{\psi}$ is well defined. The condition $[\delta+\lambda, \psi]=0$ is equivalent to the condition $D_{\bar{\delta}+\bar{\lambda}}(\bar{\psi})=0$ where $D_{\bar{\delta}+\bar{\lambda}}(\bar{\varphi})=[\bar{\delta}+\bar{\lambda}, \bar{\varphi}]$. Note that $D_{\bar{\delta}+\bar{\lambda}}^{2}=0$, so if we define the

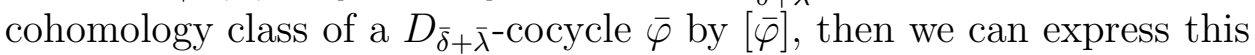
condition as $[\bar{\psi}]=0$. Moreover, if $D_{\bar{\delta}+\bar{\lambda}}(\bar{\psi})=0$, then $[\delta+\lambda, \psi]=$ $[\mu, \beta]$ for some $\beta$, but since $[\delta+\lambda, \psi] \in C^{0,3}$, which intersects the $D_{\mu^{-}}$ cobounaries trivially, this forces $[\delta+\lambda, \psi]=0$. Thus extensions are given by $D_{\bar{\delta}+\bar{\lambda}}$-cocycles.

On the other hand, if $\bar{\psi}=D_{\bar{\delta}+\bar{\lambda}}(\bar{\beta})$, then $\psi=[\delta+\lambda, \beta]$ and $[\mu, \beta]=0$. It follows that the extension determined by $\psi$ is equivalent to the one given by $\psi=0$. Thus the equivalence classes of extensions preserving the module structure $\lambda$ are parameterized by $[\bar{\psi}]$ for $\psi \in \operatorname{Hom}\left(W^{2}, M\right)$.

Theorem 4.3. The extensions of $\delta$ by $\mu$ determined by a fixed $\lambda$ satisfying

$$
[\delta, \lambda]+\frac{1}{2}[\lambda, \lambda]=0
$$

are classified by the $D_{\bar{\delta}+\bar{\lambda}}$-cohomology classes $[\bar{\psi}]$ of $D_{\bar{\delta}+\bar{\lambda}}$-cocycles $\bar{\psi}$ arising from $D_{\mu}$-cocycles $\psi \in \operatorname{Hom}\left(W^{2}, M\right)$.

When $\mu=0$, the theorem above is a reformulation, in the language of codifferentials, of the classical theorem that the equivalence classes of extensions of a Lie algebra $W$ by a module $M$ are parameterized by $H^{2}(W, M)$ (see [2]). In fact, the cohomology of a Lie algebra with coefficients in a module is determined by the coboundary operator $D_{\delta+\lambda}$, which coincides with $D_{\bar{\delta}+\bar{\lambda}}$ when $\mu=0$. 
4.3. Restricted equivalence classes of extensions. In any extension, we note that $\delta$ and $\lambda$ are $D_{\mu}$-cocycles. If $\lambda^{\prime}$ and $\lambda$ are equivalent $D_{\mu}$-cocycles and the pair $\lambda, \psi$ determines an extension, then there is an equivalent extension with $\lambda^{\prime}, \psi^{\prime}$ given by the formulas in (12). Moreover, formula (9) yields the MC formula

$$
[\bar{\delta}, \bar{\lambda}]+\frac{1}{2}[\bar{\lambda}, \bar{\lambda}]=0
$$

which means that given an representative $\lambda$ of a cohomology class $\bar{\lambda}$, there is a $\psi$ satisfying (9) precisely when $\bar{\lambda}$ satisfies the MC-equation for $\bar{\delta}$, which is a codifferential in $H_{\mu}$.

We also need $\psi$ to satisfy (11), which is not automatic. The fact that $D_{\delta+\lambda}$ anticommutes with $D_{\mu}$ implies that $D_{\mu}$ induces a coboundary operator on $\operatorname{ker}\left(D_{\delta+\lambda}\right)$. Note that $[\delta+\lambda, \delta+\lambda]$ lies in this kernel, because the triple bracket of any coderivation vanishes. As a consequence, we obtain that (9) is equivalent to the condition that $[\delta+\lambda, \delta+\lambda]$ is a $D_{\mu}$-coboundary in the restricted complex $\operatorname{ker}\left(D_{\delta+\lambda}\right)$. Moreover, even though the complex $\operatorname{ker}\left(D_{\delta+\lambda}\right)$ depends on $\lambda$, the existence of a solution to (9) depends only on the $D_{\mu}$-cohomology class of $\lambda$. Thus the assertion that $\overline{[\delta+\lambda, \delta+\lambda]}=0$ in $H_{\mu}\left(\operatorname{ker}\left(D_{\delta+\lambda}\right)\right)$ depends only on $\bar{\lambda}$, and not on the choice of a representative. However, the $\psi$ satisfying equation (9) does depend on $\lambda$. We encountered a similar situation when analyzing infinitesimal extensions, except that there, one had to consider only $H_{\mu}(\operatorname{ker}(\delta))$, instead of $H_{\mu}\left(\operatorname{ker}\left(D_{\delta+\lambda}\right)\right)$.

Now fix $\lambda$ and some $\psi$ satisfying (9). We want to characterize the set of all solutions $\psi^{\prime}=\psi+\tau$, which satisfy (9). Note that $[\mu, \tau]=0$ and

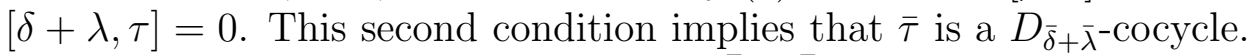
Moreover, note that $\tau \in C^{0,2}$, so that $[\bar{\delta}+\bar{\lambda}, \bar{\tau}]=0$ is equivalent to $[\mu, \tau]=0$ and $[\delta+\lambda, \tau]=0$. On the other hand, if $\bar{\tau}=[\bar{\delta}+\bar{\lambda}, \bar{\beta}]$, then $[\delta+\lambda, \beta]=0$ and $[\mu, \beta]=0$, and it is immediate that the extensions given by $\lambda, \psi$ and $\lambda, \psi+\tau$ are equivalent. Thus $D_{\bar{\delta}+\bar{\lambda}}$-coboundaries correspond to trivial extensions. As a consequence, the equivalence classes of nonequivalent extensions are parameterized by $H_{\mu, \delta+\lambda}$. Let us denote the cohomology class in $H_{\mu, \delta+\lambda}$ of a $D_{\bar{\delta}+\bar{\lambda}}$-cocycle $\bar{\varphi}$ by $[\bar{\varphi}]$. Note that $H_{\mu, \delta+\lambda}$ is naturally a graded Lie algebra. We have shown the following theorem.

Theorem 4.4. The equivalence classes of extensions of the Lie algebra structure $\delta$ on $W$ by a Lie algebra structure $\mu$ on $M$ under the action of the restricted group of automorphisms $G_{\text {rest }}$ are completely classified by the set of $D_{\mu}$-cohomology classes $\bar{\lambda}$ arising from $\lambda \in \operatorname{Hom}(M W, M)$ which satisfy the $M C$-equation

$$
\overline{[\delta+\lambda, \delta+\lambda]}=0 \in H_{\mu}\left(\operatorname{ker}\left(D_{\delta+\lambda}\right)\right)
$$


together with the $D_{\bar{\delta}+\bar{\lambda}}$-cohomology classes $[\bar{\tau}]$ of $D_{\bar{\delta}+\bar{\lambda}}$-cocycles $\bar{\tau}$ of $D_{\mu}$-cocycles $\tau \in \operatorname{Hom}\left(W^{2}, M\right)$.

4.4. General Equivalence Classes of Extensions. In the standard construction of equivalence of extensions, we have assumed that the homomorphism $f: V \rightarrow V$ acts as the identity on $M$ and $W$. We could consider a more general commutative diagram of the form

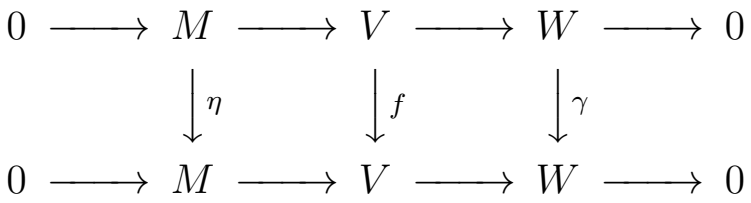

where $\eta$ and $\gamma$ are isomorphisms. It is easy to see that under this circumstance, if $d^{\prime}$ is the codifferential on the top line, and $d$ is the one below, then $\eta^{*}(\mu)=\mu^{\prime}$ and $\gamma^{*}(\delta)=\delta^{\prime}$. Therefore, if one is interested in studying the most general moduli space of all possible extensions of all codifferentials on $M$ and $W$, where equivalence of elements is given by diagrams above, then for two extensions to be equivalent, $\mu^{\prime}$ must be equivalent to $\mu$ as a codifferential on $M$, and $\delta^{\prime}$ must be equivalent to $\delta$ as a codifferential on $W$, with respect to the action of the automorphism group $\mathbf{G L}(M)$ on $M$ and $\mathbf{G L}(W)$ on $W$.

Thus, in classifying the elements of the moduli space, we first have to consider equivalence classes of codifferentials on $M$ and $W$. As a consequence, after making such a choice, we need only consider diagrams which preserve $\mu$ and $\delta$; in other words, we can assume that $\eta^{*}(\mu)=\mu$ and that $\gamma^{*}(\delta)=\delta$.

Next note that we can always decompose a general extension diagram into one of the form

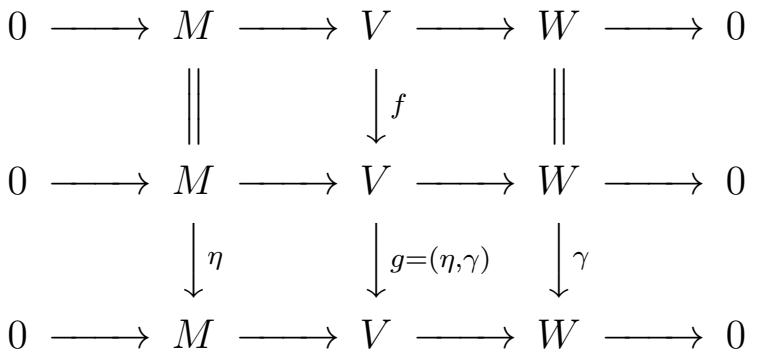

where $f=\exp (\beta)$, and $g=(\eta, \gamma)$ is an element of the group $G_{\Delta}$ consisting of block diagonal matrices. The group $G_{\text {gen }}$ of general equivalences is just the group of block upper triangular matrices, and is the semidirect product of $G_{\text {rest }}$ with $G_{\Delta}$; that is, $G_{\text {gen }}=G_{\text {rest }} \rtimes G_{\Delta}$. In fact, if $g \in G_{\Delta}$, then $g^{-1} \exp (\beta) g=\exp \left(g^{*}(\beta)\right)$.

The group $G_{\Delta}$ acts in a simple manner on cochains. If $g \in G_{\Delta}$, then $g^{*}\left(C^{k, l}\right) \subseteq C^{k, l}$ and $g^{*}\left(C^{k}\right) \subseteq C^{k}$. Since $g^{*} D_{\mu}=D_{g^{*}(\mu)} g^{*}$, the action 
induces a map

$$
g^{*}: H_{\mu} \rightarrow H_{g^{*}(\mu)},
$$

given by $g^{*}(\bar{\varphi})=\overline{g^{*}(\varphi)}$. Similarly, $g^{*} D_{\delta+\lambda}=D_{g^{*}(\delta)+g^{*}(\lambda)} g^{*}$, so we obtain a map

given by $g([\bar{\varphi}])=\left[\overline{g^{*}(\varphi)}\right]$.

$$
g^{*}: H_{\mu, \delta+\lambda} \rightarrow H_{g^{*}(\mu), g^{*}(\delta)+g^{*}(\lambda)},
$$

Let $G_{\Delta}(\mu, \delta)$ be the subgroup of $G_{\Delta}$ consisting of those elements $g$ satisfying $g^{*}(\mu)=\mu$ and $g^{*}(\delta)=\delta$. Then $G_{\Delta}(\mu, \delta)$ acts on $H_{\mu}$, and induces a map $H_{\mu, \delta+\lambda} \rightarrow H_{\mu, \delta+g^{*}(\lambda)}$. Let $G_{\Delta}(\mu, \delta, \lambda)$ be the subgroup of $g$ in $G_{\Delta}(\mu, \delta)$ such that $g^{*}(\lambda)=\lambda$. Thus $G_{\Delta}(\mu, \delta, \lambda)$ acts on both on $H_{\mu}$ and $H_{\mu, \delta+\lambda}$.

It is easy to study the behaviour of elements in $G_{\Delta}(\mu, \delta)$ on extensions. If $\lambda$ gives an extension and $g \in G_{\Delta}(\mu, \delta)$, then any element $\lambda^{\prime} \in g^{*}(\bar{\lambda})$ will determine an equivalent extension, and thus equivalence classes of $\bar{\lambda}$ under the action of the group $G_{\Delta}(\mu, \delta)$ correspond to equivalent extensions.

Now suppose that $\lambda, \psi$ gives an extension, and $\bar{\tau}$ is a $D_{\bar{\delta}+\bar{\lambda}}$-cocycle. If $g \in G_{\Delta}(\mu, \delta, \lambda)$, then

$$
g^{*}(\psi+\tau)=\psi+g^{*}(\psi)-\psi+g^{*}(\tau),
$$

so that

$$
[\bar{\tau}] \mapsto\left[\overline{g^{*}(\psi)-\psi+g^{*}(\tau)}\right]
$$

determines an action of $G_{\Delta}(\mu, \delta, \lambda)$ on $H_{\mu, \delta+\lambda}$ whose equivalence classes determine equivalent representations.

To understand the action of $G_{\text {gen }}$ on extensions, first note that any element $h \in G_{\text {gen }}$ can be expressed uniquely in the form $h=g \exp (\beta)$ where $g \in G_{\Delta}$. If $d^{\prime}=h^{*}(d)$, for an extension $d$, then we compute the components of the extension $d^{\prime}$ as follows.

$$
\begin{aligned}
\delta^{\prime} & =g^{*}(\delta) \\
\mu^{\prime} & =g^{*}(\mu) \\
\lambda^{\prime} & =g^{*}(\lambda)+\left[\mu^{\prime}, \beta\right] \\
\psi^{\prime} & =g^{*}(\psi)+\left[\delta^{\prime}+\lambda^{\prime}-\frac{1}{2}\left[\mu^{\prime}, \beta\right], \beta\right] .
\end{aligned}
$$

Clearly, $\delta^{\prime}=\delta$ and $\mu^{\prime}=\mu$ precisely when $g \in G_{\Delta}(\mu, \delta)$. Define the group $G_{\text {gen }}(\mu, \delta)$ to be the subgroup of $G_{\text {gen }}$ consisting of those $h=g \exp (\beta)$ such that $g^{*}(\delta)=\delta$ and $g^{*}(\mu)=\mu$. We have a simple decomposition $G_{\text {gen }}(\mu, \delta)=G_{\Delta}(\mu, \delta) \rtimes G_{\text {rest }}$.

Define $G_{\text {gen }}(\mu, \delta, \lambda)$ to be the subgroup of $G_{\text {gen }}(\mu, \delta)$ consisting of those $h$ such that $\lambda=g^{*}(\lambda)+[\mu, \beta]$, then $G_{\text {gen }}(\mu, \delta, \lambda)$ does not have a a simple decomposition in terms of $G_{\Delta}(\mu, \delta, \lambda)$, because the condition 
$\lambda^{\prime}=\lambda$ does not force $g \in G_{\Delta}(\mu, \delta, \lambda)$. However, we can still define an action of $G_{\text {gen }}(\mu, \delta, \lambda)$ on $H_{\mu, \delta+\lambda}^{0,2}$ by

$$
[\bar{\tau}] \rightarrow\left[\overline{g^{*}(\psi)-\psi+g^{*}(\tau)+\left[\delta+\lambda-\frac{1}{2}[\mu, \beta], \beta\right]}\right]
$$

whose equivalence classes determine equivalent representations. Note that for any element $\varphi$ in $C^{0,2}, g^{*}(\varphi)=h^{*}(\varphi)$, so we can use $h$ in place of $g$ in the formula above.

Theorem 4.5. The equivalence classes of extensions of $W$ by $M$ under the action of the group $G_{\text {gen }}$ are classified by the following data:

(1) Equivalence classes of codifferentials $\delta$ on $W$ under the action $\mathbf{G L}(W)$.

(2) Equivalence classes of codifferentials $\mu$ on $M$ under the action of the group $\mathbf{G L}(M)$.

(3) Equivalence classes of $D_{\mu}$-cohomology classes $\bar{\lambda} \in H_{\mu}^{1,1}$ which satisfy the $M C$-equation

$$
\overline{[\delta+\lambda, \delta+\lambda]}=0 \in H_{\mu}^{1,2}\left(\operatorname{ker}\left(D_{\delta+\lambda}\right)\right)
$$

under the action of the group $G_{\Delta}(\mu, \delta)$ on $H_{\mu}$.

(4) Equivalence classes of $D_{\bar{\delta}+\bar{\lambda}}$-cohomology classes $[\bar{\tau}] \in H_{\mu, \delta+\lambda}^{0,2}$ under the action of the group $G_{g e n}(\mu, \delta, \lambda)$.

We are more interested in the moduli space of extensions of $W$ by $M$ preserving fixed codifferentials on these spaces.

Theorem 4.6. The equivalence classes of extensions of a codifferential $\delta$ on $W$ by a codifferential $\mu$ on $M$ under the action of the group $G_{\text {gen }}(\mu, \delta)$ are classified by the following data:

(1) Equivalence classes of $D_{\mu}$-cohomology classes $\bar{\lambda} \in H_{\mu}^{1,1}$ which satisfy the $M C$-equation

$$
\overline{[\delta+\lambda, \delta+\lambda]}=0 \in H_{\mu}^{1,2}\left(\operatorname{ker}\left(D_{\delta+\lambda}\right)\right)
$$

under the action of the group $G_{\Delta}(\mu, \delta)$ on $H_{\mu}$.

(2) Equivalence classes of $D_{\bar{\delta}+\bar{\lambda}}$-cohomology classes $[\bar{\tau}] \in H_{\mu, \delta+\lambda}^{0,2}$ under the action of the group $G_{g e n}(\mu, \delta, \lambda)$.

To illustrate why this more general notion of equivalence is useful, we give some simple examples of extensions of ordinary and super Lie algebras. 
4.5. Simple examples of (super) Lie algebra extensions. We first study the simplest possible cases of extensions, where $W$ and $M$ are both 1-dimensional. However, we also have to take into account whether the basis elements are even or odd, which means that we obtain 3 different nontrivial cases, according to whether both $W$ and $M$ have odd bases, corresponding to the ordinary Lie algebra situation, or whether one of these spaces has an odd basis. (If both spaces have even bases, then there are no nontrivial codifferentials.) The only possible codifferentials on these spaces are the trivial ones $\delta=0$ and $\mu=0$.

4.5.1. Extending a 0|1-dimensional space by a 0|1-dimensional space. Recall that there is only 1 nontrivial Lie algebra structure on a $0 \mid 2$ dimensional space $V=\left\langle v_{1}, v_{2}\right\rangle$, given by the codifferential $\psi_{2}^{11}$. Let $M=\left\langle v_{1}\right\rangle$ and $W=\left\langle v_{2}\right\rangle$. Also, since $W^{2}=0$, the cocycle $\psi$ must vanish. The coderivation $\lambda=a \psi_{1}^{12}$ determines an extension for every value of $a$. If one considers only restricted equivalences, then the extensions for different values of $a$ are not equivalent, but if one considers the general notion of equivalence, then the codifferentials associated to nonzero values of $a$ are equivalent. Note that the moduli space of equivalence classes of codifferentials on $V$ consists of only one point, corresponding to the codifferential $\psi_{1}^{12}$. Thus the general notion of equivalence gives a more natural connection with this moduli space. Note that not every codifferential on $V$ arises from an extension of this type, but rather the equivalence classes correspond.

4.5.2. Extensions of a 0|1-dimensional by a 1|0-dimensional space. Let $M=\left\langle v_{1}\right\rangle$ and $W=\left\langle v_{2}\right\rangle$. Then any nontrivial extension is equivalent to the extension given by $\lambda=\psi_{1}^{12}$, and the cocycle $\psi$ must vanish.

4.5.3. Extensions of a 1|0-dimensional by a 0|1-dimensional space. Let $M=\left\langle v_{2}\right\rangle$ and $W=\left\langle v_{1}\right\rangle$. (Note that we change the order of the basis to conform with the principle of listing the even basis element of the space $V=\left\langle v_{1}, v_{2}\right\rangle$ first.) This time, the module structure $\lambda$ must vanish, but we do have a nontrivial cocycle $\psi$ which is always equivalent to $\psi=\psi_{2}^{11}$.

Let us compare the two moduli spaces we have just studied with the moduli space of 1|1-dimensional super Lie algebras. If $d$ is an odd element of $\operatorname{Hom}\left(V^{2}, V\right)$, then $d=a \psi_{1}^{12}+b \psi_{2}^{11}$. However, $[d, d]=0$ precisely when $a=0$ or $b=0$. Thus, the moduli space consists of exactly two elements, corresponding to the union of the two moduli spaces of extensions.

4.5.4. The moduli space of 3-dimensional Lie algebras. Let $V$ be a 3dimensional space with a completely odd basis, so the moduli space of codifferentials on this space corresponds to the moduli space of ordinary 
3-dimensional Lie algebras. We recall the decomposition of this moduli space into equivalence classes represented by the codifferentials below as given in [?].

$$
\begin{aligned}
d_{1} & =\psi_{1}^{23} \\
d_{2} & =\psi_{1}^{13}+\psi_{2}^{23} \\
d_{3} & =\psi_{3}^{12}+\psi_{2}^{13}+\psi_{1}^{23} \\
d(\lambda: \mu) & =\psi_{1}^{13} \lambda+\psi_{2}^{23} \mu+\psi_{1}^{23}
\end{aligned}
$$

The codifferential $d_{3}$ gives the simple Lie algebra $\mathfrak{s l}_{2}(\mathbb{C})$, which is not given by an extension. The other codifferentials all arise from extensions of the trivial 1-dimensional Lie algebra by a 2-dimensional Lie algebra, as well as by extensions of a 2-dimensional Lie algebra by the trivial Lie algebra. We analyze the resulting moduli spaces.

4.5.5. Extensions of a $0 \mid 2$-dimensional by a 0|1-dimensional space. Let $W=\left\langle v_{1}, v_{2}\right\rangle$ and $M=\left\langle v_{3}\right\rangle$. On $M$, there is only one codifferential, $\mu=0$. There are two nonequivalent codifferentials on $W$, given by $\delta=\psi_{1}^{12}$ and the trivial codifferential $\delta=0$. The coderivation $\lambda$ must be of the form $\lambda=\psi_{3}^{13} a+\psi_{3}^{23} b$, and the cocycle $\psi$ must be of the form $\psi=\psi_{3}^{12} c$. For any values of $a$ and $b$, we have $[\lambda, \lambda]=0$, and $[\delta+\lambda, \psi]=0$, for both choices of $\delta$ and for any values of the parameters $a, b, c$. Note that independently of the choice of $\delta, \lambda$ gives a true module structure for any extension, because $\mu=0$. Therefore $\psi=0$ always gives an extension for any solution $\lambda$ to the MC-equations.

If $\delta=\psi_{1}^{12}$, then $[\delta, \lambda]=\psi_{3}^{123} a$, and therefore $a=0$. If $\beta=\varphi_{3}^{1} x+\varphi_{3}^{2} y$, then $[\delta+\lambda, \beta]=\psi_{3}^{12}(a y-(1+b) x)$. As a consequence, the $\tau$ term in the classification can be taken as $\tau=\psi_{3}^{12} c$. Moreover, unless $a=0$ and $b=-1$, we can always find values of $x$ and $y$ so that $\tau=[\delta+\lambda, \beta]$. Thus, except in this special case, $[\bar{\tau}]=0$, and so we can assume that $c=0$. Therefore, we can assume that $\tau=0$ unless $b=-1$. In this case, the group $G(\mu, \delta)$ of automorphisms preserving $\mu$ and $\delta$ is given by automorphisms $g$ whose matrices are of the form

$$
\left[\begin{array}{lll}
r & s & 0 \\
0 & 1 & 0 \\
0 & 0 & t
\end{array}\right],
$$

where $r, s, t$ are arbitrary parameters such that $r t \neq 0$. It is easily checked that $g^{*}(\lambda)=\lambda$ for every such $g$, so different values of $b$ give rise to distinct $D_{\mu}$-cohomology classes $\bar{\lambda}=\lambda$ (since $\left.\mu=0\right)$. Note that the MC formula is satisfied by $\lambda$, so $\lambda$ determines a $\delta$-module structure on $W$. Since $G(\delta, \mu)$ is the automorphism group of $\lambda$-preserving 
automorphisms of $V$, we have to consider the action of such an automorphism on $[\bar{\tau}]$. We compute that $g^{*}(\tau)=\tau(r / t)$, which means that when $b=-1$, when we have to consider the case $c \neq 0$, we can choose $c=1$ to represent the isomorphism class of $[\bar{\tau}]$.

Putting this all together, we obtain the following codifferentials. When $\tau=0$ we obtain the codifferential $d=\psi_{1}^{12}+\psi_{3}^{23} b \sim d(1:-b)$, unless $b=-1$, in which case we obtain the codifferential $d_{2}$. When $b=-1$ and $c=1$ we obtain the codifferential $d=\psi_{1}^{12}-\psi_{3}^{23}+\psi_{3}^{12} \sim d(1: 1)$. Note that the extensions of $\delta$ are never equivalent to $d_{1}$.

Consider now the case when $\delta=0$. In this case, $[\delta, \lambda]=0$, so neither $a$ nor $b$ is forced to vanish. The group $G(\delta, \mu)$ consists of matrices of the form

$$
\left[\begin{array}{lll}
r & s & 0 \\
p & q & 0 \\
0 & 0 & t
\end{array}\right],
$$

such that $t(r q-s p) \neq 0$. In this case $g^{*}(\lambda)=\lambda=\psi_{3}^{13}(a r+b p)+$ $\psi_{3}^{23}(a s+b q)$. Thus, there are 2 isomorphism classes for $\lambda$, represented by $\lambda=\psi_{3}^{23}$ and $\lambda=0$. Again, let $\beta=\varphi_{3}^{1} x+\varphi_{3}^{2} y$. For $\lambda=\psi_{3}^{23}$, we have $[\delta+\lambda, \tau]=-\psi_{3}^{12} x$, so $\bar{\tau}$ is a $D_{\bar{\delta}+\bar{\lambda}}$-coboundary. Thus, we do not have to consider the action of the group of $\lambda$-preserving automorphisms. Thus we have only one codifferential to consider $d=\psi_{3}^{23} \sim d(0: 1)$.

For $\lambda=0$, we cannot assume that $\tau=0$. The group of $\lambda$-preserving automorphisms is just $G(\delta, \mu)$, we have two isomorphism classes for $\tau$, $\tau=0$ and $\tau=\psi_{3}^{12}$. If $\tau=0$, then $d=0$. Otherwise, $d=\psi_{3}^{12}$, which is equivalent to the codifferential $d_{1}$. This was the one case which did not show up for $\delta=\psi_{1}^{12}$.

4.5.6. Extensions of 0|1-dimensional by 0|2-dimensional spaces. In [5], the moduli space of 3 -dimensional Lie algebras was constructed by considering extensions of $\mathbb{C}$ by a 2-dimensional Lie algebra, in other words, by exactly the consideration we now present, although from a slightly different point of view. The calculations we present here are given in more detail in [5, ?]. Let $V=\left\langle v_{1}, v_{2}\right\rangle$ and $W=\left\langle v_{3}\right\rangle$. Since $W$ is 1-dimensional $\delta=\psi=0$. We must have $\lambda=\psi_{1}^{13} a+\psi_{1}^{23} b+\psi_{2}^{13} x+\psi_{2}^{23} y$ for some values of the parameters $a, b, x, y$. In all cases $[\lambda, \lambda]=0$, so $\lambda$ determines a true module structure. There are, up to isomorphism, two possible cases, $\mu=\psi_{1}^{12}$ and $\mu=0$.

Consider the case when $\mu=\psi_{1}^{12}$. In this case, the condition $[\mu, \lambda]=0$ forces $x=y=0$. Moreover, if $\beta=\varphi_{1}^{3} s+\varphi_{2}^{3} t$, then $[\mu, \beta]=\psi_{1}^{13} t-$ $\psi_{1}^{23} s$, which means that $\lambda$ is always a coboundary, so we need only consider the case $\lambda=0$. But then the extension is simply given by the 
codifferential $\mu$, which is equivalent to the codifferential $d(0: 1)$. The case $\mu=0$ gives no condition on $\lambda$, so we need only consider the action of the group $G(\mu, \delta)$ on $\lambda$. This action produces precisely the similarity classes (up to multiples) of the $2 \times 2$ matrices $\left[\begin{array}{cc}a & b \\ x & y\end{array}\right]$. This is exactly the characterization of the codifferentials $d_{1}, d_{2}$, and $d(\lambda: \mu)$, which are given by the matrices $\left[\begin{array}{ll}0 & 1 \\ 0 & 0\end{array}\right],\left[\begin{array}{ll}1 & 0 \\ 0 & 1\end{array}\right]$, and $\left[\begin{array}{ll}\lambda & 1 \\ 0 & \mu\end{array}\right]$, respectively. Thus every codifferential on $V$ except $d_{3}$ arises as an extension of $\delta=0$ by $\mu=0$.

4.5.7. A more complicated extension. In order to illustrate this construction with a more interesting example, we would like to consider an case where neither $\delta$ nor $\lambda$ vanish, which is not possible if the total space has dimension 3 . We give an example on a space of total dimension 5 .

Let $M=\left\langle v_{1}, v_{2}, v_{3}\right\rangle$ and $W=\left\langle v_{4}, v_{5}\right\rangle$. Let $\mu=d(0: 1)=\psi_{1}^{23}+$ $\psi_{2}^{23}$ and $\delta=\psi_{4}^{45}$. If we denote the $5 \times 10$ matrix of the extended codifferential by $A=\left(a_{i j}\right)$, then $\lambda$ is determined by the submatrix

$$
L=\left[\begin{array}{llllll}
a_{14} & a_{15} & a_{16} & a_{17} & a_{18} & a_{19} \\
a_{24} & a_{25} & a_{26} & a_{27} & a_{28} & a_{29} \\
a_{34} & a_{35} & a_{36} & a_{37} & a_{38} & a_{39}
\end{array}\right] .
$$

Taking into account the condition $[\mu, \lambda]=0$ yields a much simpler matrix for $\lambda$ :

$$
L=\left[\begin{array}{cccccc}
a_{14} & a_{15} & a_{16} & a_{17} & a_{18} & a_{19} \\
0 & a_{14}+a_{15} & a_{26} & 0 & a_{17}+a_{18} & a_{29} \\
0 & 0 & 0 & 0 & 0 & 0
\end{array}\right]
$$

Let $\beta=\varphi_{1}^{4} c_{11}+\varphi_{2}^{4} c_{21}+\varphi_{3}^{4} c_{31}+\varphi_{1}^{5} c_{12}+\varphi_{2}^{5} c_{22}+\varphi_{3}^{5} c_{32} \in \operatorname{Hom}(W, M)$. Then the matrix corresponding to $[\mu, \beta]$ is

$$
\left[\begin{array}{cccccc}
0 & c_{31} & -c_{21} & 0 & c_{32} & -c_{22} \\
0 & c_{31} & -c_{21} & 0 & c_{32} & -c_{22} \\
0 & 0 & 0 & 0 & 0 & 0
\end{array}\right]
$$

so by adding a $D_{\mu}$-coboundary to $\lambda$, we reduce to the case when

$$
L=\left[\begin{array}{cccccc}
a_{14} & 0 & a_{16} & a_{17} & 0 & a_{19} \\
0 & a_{14} & 0 & 0 & a_{17} & 0 \\
0 & 0 & 0 & 0 & 0 & 0
\end{array}\right]
$$

If we express $\psi$ in the form $\psi=\psi_{1}^{45} b_{1}+\psi_{2}^{45} b_{2}+\psi_{3}^{45} b_{3}$, then the condition $[\delta, \lambda]+\frac{1}{2}[\lambda, \lambda]+[\mu, \psi]=0$ gives $b_{2}=b_{3}=0, a_{14}=0$ and either $a_{17}=1$ or $a_{16}=0$. Note that after taking these conditions into account, we have $[\mu, \psi]=0$, so that actually, $\lambda$ determines a true module structure on $M$. Moreover, this means that we can choose $\psi=0$ in satisfying 
the MC-equation, and that therefore the cochain $\tau$ in the theorem is just $\tau=\psi_{1}^{45} b_{1}$. It is easily checked that $[\delta+\lambda, \tau]=0$. Moreover

$$
[\delta+\lambda, \beta]=\psi_{1}^{45}\left(c_{31} a_{19}-c_{32} a_{16}\right)-\psi_{3}^{45} c_{31},
$$

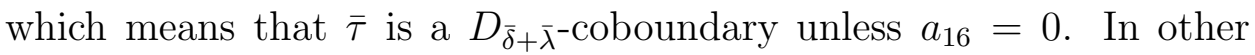
words, unless $a_{16}=0$, the extension is a semi-direct product.

Let us now assume that $a_{16}=0$. Thus $\lambda=\psi_{1}^{15}+\psi_{2}^{25}=\psi_{1}^{35} a_{19}$ and $\tau=\psi_{1}^{45} b_{1}$. We next have to take into account the action of $G(\mu, \delta)$ on the equivalence classes of $\bar{\lambda}$. It is easy to show that an element $g$ in this group has matrix given by

$$
\left[\begin{array}{ccccc}
g_{11} & g_{21}-g_{11} & g_{13} & 0 & 0 \\
0 & g_{22} & g_{23} & 0 & 0 \\
0 & 0 & 1 & 0 & 0 \\
0 & 0 & 0 & g_{44} & g_{45} \\
0 & 0 & 0 & 0 & 1
\end{array}\right] .
$$

One calculates that the matrix of $g^{*}(\lambda)$ is

$$
\left[\begin{array}{cccccc}
0 & 0 & 0 & 1 & 0 & \frac{g_{1,3} g_{2,2}-g_{2,3} g_{2,2}+g_{2,3} g_{1,1}+a_{1,9} g_{2,2}}{g_{2,2} g_{1,1}} \\
0 & 0 & 0 & 0 & 1 & \frac{g_{2,3}}{g_{2,2}} \\
0 & 0 & 0 & 0 & 0 & 0
\end{array}\right]
$$

Since the matrix of $\lambda$ is

$$
L=\left[\begin{array}{cccccc}
0 & 0 & 0 & 1 & 0 & a_{19} \\
0 & 0 & 0 & 0 & 1 & 0 \\
0 & 0 & 0 & 0 & 0 & 0
\end{array}\right],
$$

it follows that $g^{*}(\lambda)$ lies in the same $D_{\mu}$-cohomology class as $\lambda$ precisely when $g_{23}=a_{19}\left(1-g_{11}\right)+g_{13}$. However, $g^{*}(\tau)=\tau \frac{g_{44}}{g_{11}}$, which means that if $b_{1} \neq 0$, we can transform it to $b_{1}=1$. This means that there is an extension for which the cohomology class $[\bar{\tau}]$ cannot be transformed to the zero cohomology class, which is therefore an extension of $\delta$ by $\mu$ which is not given by a semi-direct product.

\section{INFINITESIMAL DEFORMATIONS OF EXTENSIONS OF LIE ALGEBRAS}

A natural question that arises when studying the moduli spaces arising from extensions is how to fit the moduli together as a space, and to answer that question, one needs to have a notion of how to move around in the moduli space. This notion is precisely the idea of deformations, in this case, deformations of the extensions. We will classify the infinitesimal deformations of an extension. 
Suppose that $d=\delta+\mu+\lambda+\psi$ is an extension, and we consider the infinitesimal deformation of this extension

$$
d_{t}=d+t(\eta+\zeta)
$$

where $\eta \in \operatorname{Hom}(M W, M)$ represents a deformation of the $\lambda$ structure, and $\zeta \in \operatorname{Hom}\left(W^{2}, M\right)$ gives a deformation of the $\psi$ structure. Here, we don't consider deformations which involve deforming the $\delta$ or $\mu$ structure. The infinitesimal condition is that $t^{2}=0$, in which case, as usual, the condition for $\eta, \zeta$ to determine a deformation is, infinitesimally, that $[d, \eta+\zeta]=0$. We split this one condition up into the four conditions below.

$$
\begin{aligned}
& {[\delta+\lambda, \eta]+[\mu, \zeta]=0} \\
& {[\delta+\lambda, \zeta]+[\psi, \eta]=0} \\
& {[\mu, \eta]=0} \\
& {[\psi, \zeta]=0}
\end{aligned}
$$

These conditions are symmetric in the roles of $\psi$ and $\mu$, but as in the case of infinitesimal extensions, this symmetry is a bit misleading. For example the condition (19) is automatic for $\zeta \in \operatorname{Hom}\left(W^{2}, M\right)$, but condition (18) is not automatic for $\eta \in \operatorname{Hom}(M W, M)$.

Note that since $\eta$ is a $D_{\mu}$-cocycle, $\bar{\eta}$ is well defined, and the first of these equations implies that $\bar{\eta}$ is a $D_{\bar{\delta}+\bar{\lambda}}$-cocycle. Since $[\psi, \psi]=$ 0 , it determines a coboundary operator $D_{\psi}$ as well. Denote the $D_{\psi^{-}}$ cohomology class of a $D_{\psi}$-cocycle $\varphi$ by $\overline{\bar{\varphi}}$ and the set of cohomology classes by $H_{\psi}$. Note that $H_{\psi}$ inherits the structure of a Lie algebra.

Since $[\delta+\lambda, \psi]=0$, it follows that $\overline{\overline{\delta+\lambda}}$ is well defined. Moreover, we have

$$
\begin{aligned}
{[\overline{\overline{\delta+\lambda}},[\overline{\overline{\delta+\lambda}}, \overline{\bar{\varphi}}]] } & =\overline{\overline{[\delta+\lambda,[\delta+\lambda, \varphi]]}}=\overline{\overline{\left[\frac{1}{2}[\delta+\lambda, \delta+\lambda], \varphi\right]}} \\
& =-\overline{\overline{[[\mu, \psi], \varphi]}}=-\overline{\overline{[\mu,[\psi, \varphi]]}}=0,
\end{aligned}
$$

so $D \overline{\overline{\delta+\lambda}}$ is a differential on $H_{\psi}$. Denote the cohomology class of a $D \overline{\overline{\delta+\lambda}}$ cocycle $\overline{\bar{\varphi}}$ by $[\overline{\bar{\varphi}}]$ and the set of cohomology classes by $H_{\psi, \delta+\lambda}$. Note that $H_{\psi, \delta+\lambda}$ inherits the structure of a Lie algebra.

We first remark that conditions (16) and (18) imply that $[\bar{\eta}]$ is well defined, and (17) and (19) imply that $[\overline{\bar{\zeta}}]$ is well defined.

Next we introduce an action of $D_{\psi}$ on $H_{\mu, \delta+\lambda}$. It is not possible to extend the operation of bracketing with $\psi$ to the $D_{\mu}$-cohomology, because $[\mu, \psi] \neq 0$. Moreover, even if $[\mu, \varphi]=0$, it does not follow that $[\mu,[\psi, \varphi]]=0$. However, we can extend the bracket to $H_{\mu, \delta+\lambda}$ as 
follows. A cohomology class $[\bar{\varphi}]$, is given by a $\varphi$ such that $[\mu, \varphi]=0$ and $[\delta+\lambda, \varphi]=[\mu, \beta]$ for some $\beta$. Note that

$$
\begin{aligned}
{[\mu,[\psi, \varphi]] } & =[[\mu, \psi], \varphi]=-[\delta+\lambda,[\delta+\lambda, \varphi]] \\
& =-[\delta+\lambda,[\mu, \beta]]=[\mu,[\delta+\lambda, \beta]] .
\end{aligned}
$$

This suggests that it might be possible to define a map by

$$
D_{\psi}([\bar{\varphi}])=[\overline{[\psi, \varphi]-[\delta+\lambda, \beta]}],
$$

where $\beta$ is any solution to $[\delta+\lambda, \varphi]=[\mu, \beta]$. There are a few things we have to check in order to see that this is a well-defined action.

(1) Show that $[\bar{\delta}+\bar{\lambda}, \overline{[\psi, \varphi]-[\delta+\lambda, \beta]}]=0$. This follows from

$$
\begin{aligned}
{[\delta+\lambda,[\psi, \varphi]-[\delta+\lambda, \beta]] } & =[\delta+\lambda,[\psi, \varphi]]-[\delta+\lambda,[\delta+\lambda, \beta]] \\
& =-[\psi,[\delta+\lambda, \varphi]]+[[\mu, \psi], \beta] \\
& =-[\psi,[\mu, \beta]]+[[\mu, \psi], \beta]=[\mu,[\psi, \beta]]
\end{aligned}
$$

This first step has established that $[\overline{[\psi, \varphi]-[\delta+\lambda, \beta]}]$ is at least well-defined.

(2) Show that, given a $\varphi$, the formula does not depend on the choice of $\beta$. Suppose that $[\delta+\lambda, \varphi]=\left[\mu, \beta^{\prime}\right]$. Then $\left[\mu, \beta^{\prime}-\beta\right]=0$

$$
\begin{aligned}
& {\left.\left[\overline{[\psi, \varphi]-[\delta+\lambda, \beta]}-\overline{[\psi, \varphi]-\left[\delta+\lambda, \beta^{\prime}\right.}\right]\right] } \\
= & {\left[\overline{\left[\delta+\lambda, \beta^{\prime}-\beta\right]}\right]=\left[\left[\bar{\delta}+\bar{\lambda}, \overline{\beta^{\prime}-\beta}\right]\right]=0, }
\end{aligned}
$$

because $\bar{\delta}+\bar{\lambda}$ is a differential on $H_{\mu}$.

(3) Show that if $[\bar{\varphi}]=0$, then $[\overline{[\psi, \varphi]-[\delta+\lambda, \beta]}]=0$. If $[\bar{\varphi}]=0$, then we can express $\varphi=[\hat{\delta}+\lambda, \alpha]+[\mu, \gamma]$ for some $\alpha$ and $\gamma$ such that $[\mu, \alpha]=0$. Now

$$
\begin{aligned}
{[\psi, \varphi]-[\delta+\lambda, \beta] } & =[\psi,[\delta+\lambda, \alpha]+[\mu, \gamma]]-[\delta+\lambda, \beta] \\
& =-[\delta+\lambda,[\psi, \alpha]+\beta]+[\psi,[\mu, \gamma] \\
& =-[\delta+\lambda,[\psi, \alpha]+\beta]+[[\psi, \mu], \gamma]-[\mu,[\psi, \gamma]] \\
& =-[\delta+\lambda,[\psi, \alpha]+\beta]-[\delta+\lambda,[\delta+\lambda, \gamma]]-[\mu[\psi, \gamma]] \\
& =-[\delta+\lambda,[\psi, \alpha]+\beta+[\delta+\lambda, \gamma]]-[\mu,[\psi, \gamma]] .
\end{aligned}
$$

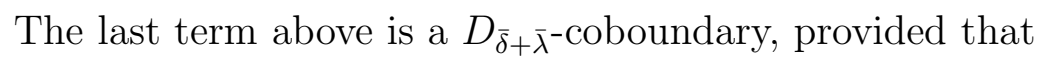

$$
[\mu,[\psi, \alpha]+\beta+[\delta+\lambda, \gamma]]=0
$$


But

$$
\begin{aligned}
{[\mu,[\psi, \alpha]+\beta+[\delta+\lambda, \gamma]] } & =-[\mu,[\psi, \alpha]]+[\mu, \beta]+[\mu,[\delta+\lambda, \gamma]] \\
& =-[\delta+\lambda,[\delta+\lambda, \alpha]]+[\delta+\lambda, \varphi]-[\delta+\lambda,[\mu, \gamma]] \\
& =[\delta+\lambda,-[\delta+\lambda, \alpha]+\varphi-[\mu, \gamma]]=0 .
\end{aligned}
$$

It is straightforward to verify that $D_{\psi}$ is actually a Lie algebra morphism on the graded Lie algebra $H_{\mu, \delta+\lambda}$, and that $D_{\psi}^{2}=0$. Let us denote the cohomology by $H_{\mu, \delta+\lambda, \psi}$, and the $D_{\psi}$-cohomology class of a $D_{\psi}$-cocycle $[\bar{\varphi}]$ by $\{[\bar{\varphi}]\}$.

In a very similar manner, one can show that one can define $D_{\mu}$ on $H_{\psi, \delta+\lambda}$ by

$$
D_{\mu}([\overline{\bar{\varphi}}])=[\overline{\overline{[\mu, \varphi]-[\delta+\lambda, \beta]}}],
$$

where $\beta$ is any coderivation satisfying $[\delta+\lambda, \varphi]=[\psi, \beta]$. Then $D_{\mu}$ is a Lie algebra morphism on $H_{\psi, \delta+\lambda}$ whose square is zero, and we denote the resulting cohomology by $H_{\psi, \delta+\lambda, \mu}$ and the cohomology class of a $D_{\mu}$-cocycle $[\overline{\bar{\varphi}}]$ by $\{[\overline{\bar{\varphi}}]\}$.

Thus we have constructed two different triple cohomology groups. It turns out that the first one will play a more important role in the classification of infinitesimal deformations of extensions.

Lemma 5.1. Suppose that $d=\delta+\mu+\lambda+\psi$ is an extension of the codifferentials $\delta$ on $W$ by $\mu$ on $M$, that $\eta \in \operatorname{Hom}(M W, M)$ and $\zeta \in$ $\operatorname{Hom}\left(W^{2}, M\right)$. If

$$
d_{t}=d+t(\eta+\zeta)
$$

determines an infinitesimal deformation of $d$ then

(1) $\{[\bar{\eta}]\}$ is well defined.

(2) $\{[\overline{\bar{\zeta}}]\}$ is well defined.

Proof. We already saw that if $\eta$ and $\zeta$ determine a deformation, then $[\bar{\eta}]$ and $[\overline{\bar{\zeta}}]$ are defined. Now, $D_{\psi}([\bar{\eta}])=[\overline{[\psi, \eta]-[\delta+\lambda, \beta]}]$, where $[\delta+\lambda, \eta]=[\mu, \beta]$. We obtain that

$$
[\psi, \eta]-[\delta+\lambda, \beta]=-[\delta+\lambda, \zeta]-[\delta+\lambda, \beta]=-[\delta+\lambda, \beta+\zeta] .
$$

Moreover

$$
[\mu, \beta+\zeta]=[\mu, \beta]+[\mu, \zeta]=[\delta+\lambda, \eta]-[\delta+\lambda, \eta]=0 .
$$

Thus $\overline{[\psi, \eta]-[\delta+\lambda, \beta]}=[\bar{\delta}+\bar{\lambda},-\overline{\alpha+\beta}]$, so that $[\overline{[\psi, \eta]-[\delta+\lambda, \beta]}]=$ 0 . The proof that $D_{\mu}([\overline{\bar{\zeta}}])=0$ is similar. 
Now, let us suppose that $\{[\bar{\eta}]\}$ exists. We will show that this condition alone is sufficient to guarantee the existence of a $\zeta$ such that conditions (16), (17), (18) and (19) are satisfied. Since $[\bar{\eta}]$ is defined, there is some $\beta \in \operatorname{Hom}\left(W^{2}, M\right)$ such that

$$
[\delta+\lambda, \eta]=[\mu, \beta]
$$

Note that this $\beta$ is determined up to a $D_{\mu}$-cocycle. Now,

$$
0=D_{\psi}([\bar{\eta}])=[\overline{[\psi, \eta]-[\delta+\lambda, \beta]}]
$$

SO

$$
\overline{[\psi, \eta]-[\delta+\lambda, \beta]}=[\bar{\delta}+\bar{\lambda}, \bar{\alpha}]
$$

and therefore

$$
[\psi, \eta]-[\delta+\lambda, \beta]=[\delta+\lambda, \alpha]+[\mu, \gamma] .
$$

Since $[\psi, \eta]-[\delta+\lambda, \beta] \in \operatorname{Hom}\left(W^{3}, M\right)$, the term $[\mu, \gamma]$ must vanish. Now, by construction, $[\mu, \alpha]=0$, so we observe that

$$
\begin{aligned}
{[\delta+\lambda, \eta] } & =[\mu, \beta+\alpha] \\
{[\psi, \eta] } & =[\delta+\lambda, \beta+\alpha]
\end{aligned}
$$

But this means that $\zeta=-(\beta+\alpha)$ satisfies conditions (16) and (17), and thus all the conditions are satisfied, since condition (18) is that $\bar{\eta}$ exists and condition (19) is automatic. In other words, we have constructed a solution to the infinitesimal deformation problem, given an arbitrary $D_{\psi}$-cocycle $[\bar{\eta}]$.

Next we ask what variation is possible in $\zeta$; in other words, given that $\zeta$ is a solution, when is $\zeta^{\prime}=\zeta+\tau$ another solution. It is easy to see that $[\mu, \tau]=0$ and $[\delta+\lambda, \tau]=0$. This means that $\bar{\tau}$ is a $D_{\bar{\delta}+\bar{\lambda}}$-cocycle, so that the cohomology class $[\bar{\tau}]$ is defined. Moreover, $[\psi, \tau]=0$ automatically, so it follows that $\{[\bar{\tau}]\}$ is defined. Note that for any $\tau \in \operatorname{Hom}\left(W^{2}, M\right),[\bar{\tau}]$ is defined precisely when the conditions $[\mu, \tau]=0$ and $[\delta+\lambda, \tau]=0$ are satisfied, and the existence of $[\bar{\tau}]$ implies the existence of $\{[\bar{\tau}]\}$.

In light of lemma $[5.1$, one might expect that $\{[\overline{\bar{\tau}}]\}$ would be the natural object of study, and indeed, the conditions on $\tau$ imply that $\{[\overline{\bar{\tau}}]\}$ exists. However, the existence of $\{[\overline{\bar{\tau}}]\}$ is not equivalent to the conditions $[\mu, \tau]=0$ and $[\delta+\lambda, \tau]=0$, so it turns out that $\{[\overline{\bar{\tau}}]\}$ is not the right object to classify the infinitesimal deformations.

To classify the infinitesimal deformations, we need to consider infinitesimal equivalences. These are given by maps $g=\exp (t(\alpha+\beta+\gamma))$, where $\alpha \in \operatorname{Hom}(M, M), \beta \in \operatorname{Hom}(W, M)$ and $\gamma \in \operatorname{Hom}(W, W)$, and 
we suppose that $t^{2}=0$. If we set $d_{t}^{\prime}=g^{*}\left(d_{t}\right)$, then $d_{t}^{\prime}=d+t\left(\eta^{\prime}+\zeta^{\prime}\right)$, where

$$
\begin{aligned}
& \eta^{\prime}=\eta+[\delta+\lambda, \alpha+\gamma]+[\mu, \beta] \\
& \zeta^{\prime}=\zeta+[\psi, \alpha+\gamma]+[\delta+\lambda, \beta] .
\end{aligned}
$$

Moreover, $[\delta, \alpha+\gamma]=[\mu, \alpha+\gamma]=0$, because we require that $g$ preserves $\delta$ and $\mu$.

Let us examine the conditions for two cohomology classes $\{[\bar{\eta}]\}$ and $\left\{\left[\overline{\eta^{\prime}}\right]\right\}$ to be equal. First, we must have

$$
\left[\overline{\eta^{\prime}}\right]=[\bar{\eta}]+D_{\psi}([\bar{\sigma}]) .
$$

However, the only way this could be possible is if $\sigma \in \operatorname{Hom}(M, W)$, which is not allowed. Thus, when $\eta \in \operatorname{Hom}(M W, M)$, the cohomology class $\{[\bar{\eta}]\}$ coincides with the cohomology class $[\bar{\eta}]$. However, the existence of $[\bar{\eta}]$ does not imply the existence of $\{[\bar{\eta}]\}$ as it did for $\tau \in \operatorname{Hom}\left(W^{2}, M\right)$. Continuing our analysis, we obtain

$$
\overline{\eta^{\prime}}=\bar{\eta}+[\bar{\delta}+\bar{\lambda}, \bar{\xi}]
$$

for some $\xi=\alpha+\gamma$, with $\alpha \in \operatorname{Hom}(M, M)$ and $\gamma \in \operatorname{Hom}(W, W)$, such that $[\mu, \alpha+\gamma]=0$. Finally, we can express

$$
\eta^{\prime}=\eta+[\delta+\lambda, \alpha+\gamma]+[\mu, \beta]
$$

where $\beta \in \operatorname{Hom}(W, M)$. But this is precisely the $\eta^{\prime}$ that arises from $g^{*}\left(d_{t}\right)$. Thus, in order for two infinitesimal deformations $d_{t}$ and $d_{t}^{\prime}$ to be equivalent, $\eta$ and $\eta^{\prime}$ must belong to the same cohomology class in $H_{\mu, \delta+\lambda, \psi}$.

Next, we can suppose that $\eta^{\prime}=\eta$, in other words, that

$$
[\delta+\lambda, \alpha+\gamma]+[\mu, \beta]=0 .
$$

This is precisely the condition that $[\overline{\alpha+\gamma}]$ exists. One should consider the action of $g^{*}$ on $d_{t}$ as follows. If the term from $\operatorname{Hom}\left(W^{2}, M\right)$ in $d_{t}$ is given by $\zeta+\tau$, then the corresponding term in $d_{t}^{\prime}$ should be $\zeta+\tau^{\prime}$. In other words, we have

$$
\tau^{\prime}=\tau+[\psi, \alpha+\gamma]+[\delta+\lambda, \beta]
$$

But this means that

$$
\left[\overline{\tau^{\prime}}\right]=[\bar{\tau}]+D_{\psi}([\overline{\alpha+\gamma}])
$$

Thus $\left\{\left[\overline{\tau^{\prime}}\right]\right\}=\left\{\left[\overline{\tau^{\prime}}\right]\right\}$.

Now, let us investigate when it is possible for $\{[\bar{\tau}]\}=\left\{\left[\overline{\tau^{\prime}}\right]\right\}$, for $\tau, \tau^{\prime} \in \operatorname{Hom}\left(W^{2}, M\right)$. First, we have

$$
\left[\overline{\tau^{\prime}}\right]=[\bar{\tau}]+D_{\psi}([\bar{\sigma}])=[\overline{\tau+[\psi, \sigma]-[\delta+\lambda, \rho]}],
$$


where

$$
[\delta+\lambda, \sigma]=[\mu, \rho]
$$

We obtain that

$$
\overline{\tau^{\prime}}=\overline{\tau+[\psi, \sigma]-[\delta+\lambda, \rho]}+[\bar{\delta}+\bar{\lambda}, \bar{\xi}]
$$

for some $\xi$. Finally, we obtain

$$
\tau^{\prime}=\tau+[\psi, \sigma]-[\delta+\lambda, \rho+\xi]+[\mu, \epsilon]
$$

However, because $\tau^{\prime} \in \operatorname{Hom}\left(W^{2}, M\right)$, we must have $[\mu, \epsilon]$. Also, since $[\mu, \xi]=0$, we have $[\delta+\lambda, \sigma]=[\mu, \rho+\xi]$, so the combined term $\rho+\xi$ plays the same role for $\sigma$ as $\rho$ does in terms of the triple coboundary operator $D_{\psi}$. If we set $\sigma=\alpha+\gamma$ and $\rho+\xi=-\beta$, then we see that $\left[\overline{\tau^{\prime}}\right]=[\bar{\tau}]+D_{\psi}([\overline{\alpha+\gamma}])$. In other words, the element $\{[\bar{\tau}]\}$ completes the classification of $d_{t}$ up to equivalence. We have shown that

Summarizing these results we obtain

Theorem 5.2. Suppose that $d=\delta+\mu+\lambda+\psi$ is an extension of the codifferentials $\delta$ on $W$ by $\mu$ on $M$.

An element $\eta \in \operatorname{Hom}(M W, M)$ gives rise to an infinitesimal deformation for some $\zeta \in \operatorname{Hom}\left(W^{2}, M\right)$ if and only if the triple cohomology class $\{[\bar{\eta}]\}$ is well defined. In this case, if $\zeta$ is any coderivation such that $\eta, \zeta$ determine an infinitesimal deformation, then $\zeta^{\prime}=\zeta+\tau$ determines another infinitesimal deformation if and only if the double cohomology class $[\bar{\tau}]$ is well defined.

Moreover the infinitesimal equivalence classes of infinitesimal deformations are classified by the triple cohomology classes $\{[\bar{\eta}]\}$ and $\{[\bar{\tau}]\}$.

\subsection{An example of an infinitesimal deformation of an exten-}

sion. Consider the extensions from the example in section 4.5.5 Recall that $\delta=\psi_{1}^{12}, \mu=0$ and $\lambda=\psi_{3}^{23} b$. Let us first consider the case when $\psi=0$. We must have $\eta=\psi_{3}^{13} r+\psi_{3}^{23} s$ for some value of the parameters $r$ and $s$. Since $\mu=0, \bar{\eta}$ is obviously well defined. Next, we have $[\delta+\lambda, \eta]=\psi_{3}^{123} r$, so in order for $[\bar{\eta}]$ to exist, $r=0$. Let

$$
\begin{aligned}
& \alpha=\varphi_{1}^{1} a_{11}+\varphi_{2}^{1} a_{12}+\varphi_{1}^{2} a_{21}+\varphi_{2}^{2} a_{22} \\
& \beta=\varphi_{3}^{1} x+\varphi_{3}^{2} y \\
& \gamma=\varphi_{3}^{3} c .
\end{aligned}
$$

Then

$$
[\delta+\lambda, \alpha+\gamma]=\psi_{1}^{12} a_{22}-\psi_{2}^{12} a_{21}+\psi_{3}^{13} a_{21} b+\psi_{3}^{23} a_{22} b
$$

from which it follows that $\bar{\eta}$ is not a $D_{\bar{\delta}+\bar{\lambda}}$-cohomology class. Since $\psi=$ $0,\{] \bar{\eta}]\}$ is defined and is not a $D_{\psi}$-coboundary. Therefore, $\{[\eta]\} \neq 0$ unless $s=0$. Next, we must have $\tau=\psi_{3}^{12} z$ for some $z$. But then $[\bar{\delta}+$ 
$\bar{\lambda}, \bar{\tau}]=0$, so $\{[\bar{\tau}]\}$ is defined. However $[\delta+\lambda, \beta]=-\psi_{3}^{12} x(1+b)$, which means that unless $b=-1, \bar{\tau}$ is a $D_{\psi}$-coboundary, and therefore $\{[\bar{\tau}]\}=$ 0 . As a consequence, when $b \neq-1$, the infinitesimal deformations are given by the $\eta$ 's alone.

Now, when $b=-1$ and $\psi=0$, we obtain a nontrivial cohomology class $\{[\bar{\tau}]\}$, which means that the infinitesimal deformations are governed by 2 parameters, $s$ and $z$. Let us consider the other nontrivial case, when $b=-1$ and $\psi=\psi_{3}^{12}$. It still turns out that $D_{\psi}(\bar{\tau})=0$, but this time, we compute $[\psi, \alpha+\gamma]=\psi_{3}^{12}\left(a_{11}+a_{22}-c\right)$. We also must have $[\delta+\lambda, \alpha+\gamma]=0$, which forces $a_{21}=a_{22}=0$, but even so, there is a solution for $\tau=[\psi, \alpha+\gamma]$. This means that $\{[\bar{\tau}]\}=0$, and like the case when $b \neq-1$, we only get one parameter for our infinitesimal deformations.

In [5, ?], the moduli space of 3-dimensional Lie algebras was studied in detail, and it was shown that codifferentials of the form $d(1: b)$ deform only along this family, but the codifferential $d_{2}$ has a jump deformation to $d(1: 1)$ as well as deformations along the family. The picture we have painted of the infinitesimal deformations of the extensions mirrors that behaviour.

In fact, there are natural notions of universal infinitesimal deformations as well as miniversal deformations of extensions, but these ideas deserve another paper.

\section{Infinitesimal Deformations of Representations}

The notion of deformations of representations has applications in physics, but is not well studied in the mathematics literature. There is a nice paper [8] which studies some aspects of these issues. In this section, we give a complete classification of infinitesimal deformations of representations of Lie algebras and Lie superalgebras.

Suppose that $M$ is a Lie algebra, with multiplication $\mu$ which is also a module over $W$. In other words, we are studying an extension of $W$ by $M$ for which the cocycle $\psi$ vanishes. There are two interesting problems we could study.

(1) Allow the module structure $\lambda$ and the algebra structure $\delta$ to vary, but keep $\mu$ fixed. This case includes the study of deformations of a module structure where the module does not have an algebra structure.

(2) Allow the module structure $\lambda$ and the multiplication $\mu$ to vary, but keep the algebra structure $\delta$ fixed.

In both of these scenarios, we think of the structures on $M$ and $W$ as

being distinct, with interaction only through $\lambda$, so when considering 
automorphisms of the structures, it is reasonable to restrict to automorphisms of $S(V)$ which do not mix the $W$ and $M$ terms, in other words, those automorphisms which are given by an automorphism of $M$ and and automorphism of $W$.

Then we have the following maps:

$$
\begin{aligned}
& D_{\delta}: C^{n} \rightarrow C^{n+1} \\
& D_{\lambda}: C^{n} \rightarrow C^{1, n} \\
& D_{\delta+\lambda}: C^{k, l} \rightarrow C^{k, l+1} \\
& D_{\mu}: C^{k, l} \rightarrow C^{k+1, l} .
\end{aligned}
$$

In the setup of this problem, we only are interested in $C^{k, l}$ for $k \geq 1$, so we shall restrict our space of cochains in this manner. Because of this restriction, we note that an element in $C^{1,1}$ can be a $D_{\mu}$-cocycle, but never a $D_{\mu^{-}}$coboundary. Moreover $C^{n} \subseteq \operatorname{ker}\left(D_{\mu}\right)$, so an element in $C^{2}$ is always a $D_{\mu}$-cocycle, and never a $D_{\mu}$-coboundary.

Because $\psi=0$, the MC-equation $[\delta, \lambda]+\frac{1}{2}[\lambda \lambda]=0$ is satisfied, so that $D_{\delta+\lambda}^{2}=0$. Since

$$
\begin{aligned}
\left(D_{\lambda} D_{\delta}+D_{\delta+\lambda} D_{\lambda}\right)(\varphi) & =[\lambda,[\delta, \varphi]]+[\delta+\lambda,[\lambda, \varphi]] \\
& =[[\lambda, \delta], \varphi]-[\delta,[\lambda, \varphi]]+[\delta,[\lambda, \varphi]]+[\lambda,[\lambda, \varphi]] \\
& =[[\delta, \lambda], \varphi]+\left[\frac{1}{2}[\lambda, \lambda], \varphi\right]=0 .
\end{aligned}
$$

we have

$$
D_{\lambda} D_{\delta}+D_{\delta+\lambda} D_{\lambda}=0 .
$$

If we denote the $D_{\mu}$-cohomology class of a $D_{\mu}$-cocycle $\varphi$ by $\bar{\varphi}$ as usual, then since $\bar{\lambda}$ and $\bar{\delta}$ are defined, we get the following version of this equation, applicable to the cohomology space $H_{\mu}$.

$$
D_{\bar{\lambda}} D_{\bar{\delta}}+D_{\bar{\delta}+\bar{\lambda}} D_{\bar{\lambda}}=0
$$

As usual, let us denote the $D_{\bar{\delta}+\bar{\lambda}}$-cohomology class of a $D_{\bar{\delta}+\bar{\lambda}}$-cocycle $\bar{\varphi}$ by $[\bar{\varphi}]$.

Let us study the first scenario, where we allow $\lambda$ and $\delta$ to vary, in other words, we consider

$$
d_{t}=d+t\left(\delta_{1}+\lambda_{1}\right)
$$

where $\delta_{1} \in C^{2}$ and $\lambda_{1} \in C^{1,1}$ represent the variations in $\delta$ and $\lambda$. The infinitesimal condition $\left[d_{t}, d_{t}\right]=0$ is equivalent to the three conditions 
for a deformation of a module structure:

$$
\begin{aligned}
{\left[\delta, \delta_{1}\right] } & =0 \\
{\left[\lambda, \delta_{1}\right]+\left[\delta+\lambda, \lambda_{1}\right] } & =0 \\
{\left[\mu, \lambda_{1}\right] } & =0 .
\end{aligned}
$$

By the third condition above $\bar{\lambda}_{1}$ is well defined, and $\bar{\delta}_{1}$ is defined. We claim that if $D_{\bar{\delta}}\left(\bar{\delta}_{1}\right)=0$, which is the first condition, then the $D_{\bar{\delta}+\bar{\lambda}}$-cohomology class $\left[D_{\bar{\lambda}}\left(\delta_{1}\right)\right]$ is well defined and depends only on the $D_{\delta}$-cohomology class of $\delta_{1}$. It is well defined because

$$
D_{\bar{\delta}+\bar{\lambda}} D_{\bar{\lambda}}\left(\bar{\delta}_{1}\right)=-D_{\bar{\lambda}} D_{\bar{\delta}}\left(\bar{\delta}_{1}\right)=0 .
$$

To see that it depends only on the $D_{\delta}$-cohomology class of $\bar{\delta}$, we apply

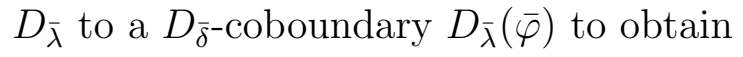

$$
D_{\bar{\lambda}} D_{\bar{\delta}}(\bar{\varphi})=D_{\bar{\delta}+\bar{\lambda}} D_{\bar{\lambda}}(-\bar{\varphi})
$$

which is a $D_{\bar{\delta}+\bar{\lambda}}$-coboundary. The second condition for a deformation of the module structure implies that $\left[D_{\bar{\lambda}}(\bar{\delta})\right]=0$. Moreover, if this statement holds, then there is some $\lambda_{1}$ such that $\delta_{1}$ and $\lambda_{1}$ determine a deformation of the module structure. We see that $\lambda_{1}^{\prime}=\lambda+\tau$ is another solution precisely when $\bar{\tau}$ exists and $D_{\bar{\delta}+\bar{\lambda}}(\bar{\tau})=0$. Thus, given one solution $\lambda_{1}$, the set of solutions is determined by the $D_{\bar{\delta}+\bar{\lambda}^{-c o c y c l e s}}$ $\tau \in C^{1,1}$.

Now let us consider infinitesimal equivalence. We suppose that $\alpha \in$ $C^{1,0}$ and $\gamma \in C^{1}$, and $g=\exp \left(t(\alpha+\beta)\right.$. If $d_{t}^{\prime}=g^{*}\left(d_{t}\right)$ is given by the cochains $\delta_{1}^{\prime}$ and $\lambda_{1}^{\prime}$, then we have

$$
\begin{aligned}
\delta_{1}^{\prime} & =\delta_{1}+D_{\delta}(\gamma) \\
\lambda_{1}^{\prime} & =\lambda_{1}+D_{\lambda}(\alpha+\gamma) \\
D_{\mu}(\alpha+\gamma) & =0 .
\end{aligned}
$$

It follows that the set of equivalence classes of deformations are determined by $D_{\delta}$ cohomology classes of $\delta_{1} \in C^{2}$. If we fix $\delta_{1}$ such that $D_{\delta}\left(\delta_{1}\right)=0$ and $\lambda_{1}$ satisfying the rest of the conditions of a deformation, then expressing $\tau^{\prime}=\tau+D_{\lambda}(\alpha+\gamma)$. But, since $D_{\delta}(\alpha+\gamma)=0$, this means we can express $\tau^{\prime}=\tau+D_{\delta+\lambda}(\alpha+\gamma)$ and $D_{\mu}(\alpha+\gamma)$, which means that $\bar{\tau}^{\prime}=\bar{\tau}+D_{\bar{\delta}+\bar{\lambda}}(\bar{\alpha}+\bar{\gamma})$, and the solutions for $\tau$ are given by

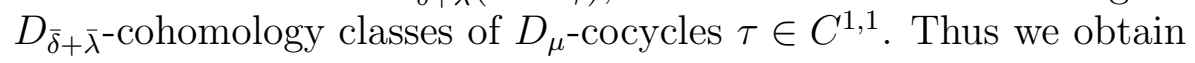

Theorem 6.1. The infinitesimal deformations of a module $M$ with multiplication $\mu$ over a Lie algebra $\delta$, allowing the algebra structure $\delta$ on $W$ and module structure $\lambda$ to vary are classified by 
(1) $D_{\delta}$-cohomology classes of $D_{\delta}$-cocycles $\delta_{1} \in C^{2}$ satisfying the condition

$$
\left[D_{\bar{\lambda}}\left(\bar{\delta}_{1}\right)\right]=0
$$

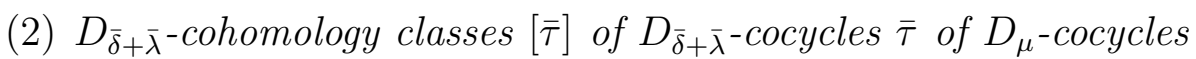
$\tau \in C^{1,1}$.

Finally, let us study the second scenario, where we allow $\lambda$ and $\mu$, but not $\delta$, to vary. We write $d_{t}=d+t\left(\lambda_{1}+\mu_{1}\right)$, where $\lambda_{1} \in C^{1,1}$ is the variation of $\lambda$ and $\mu_{1} \in C^{2,0}$ is the variation in $\mu$. The Jacobi identity $\left[d_{t}, d_{t}\right]=0$ gives three conditions for a deformation of the module structure.

$$
\begin{aligned}
& D_{\mu}\left(\mu_{1}\right)=0 \\
& D_{\delta+\lambda}\left(\mu_{1}\right)+D_{\mu}\left(\lambda_{1}\right)=0 \\
& D_{\delta+\lambda}\left(\lambda_{1}\right)=0 .
\end{aligned}
$$

Recall that $D_{\mu}$ maps $\operatorname{ker}\left(D_{\delta+\lambda}\right)$ to itself, so $H_{\mu}(\operatorname{ker}(\delta+\lambda))$ is well defined. The first condition on a deformation says that $\bar{\mu}_{1}$ is well defined. We claim that in that case, $\overline{D_{\delta+\lambda}\left(\mu_{1}\right)}$ is a well defined element of $H_{\mu}\left(\operatorname{ker}(\delta+\lambda)\right.$ which depends only on $\bar{\mu}_{1}$. This is clear, since $D_{\delta+\lambda}\left(\mu_{1}\right) \in \operatorname{ker}\left(D_{\delta+\lambda}\right)$, and $D_{\mu} D_{\delta+\lambda}\left(\mu_{1}\right)=-D_{\delta+\lambda} D_{u}\left(\mu_{1}\right)=0$. The second condition on a deformation says simply that $\overline{D_{\delta+\lambda}\left(\mu_{1}\right)}=0$, and the fact that this statement is true in $H_{\mu}(\operatorname{ker}(\delta+\lambda))$ is the third condition. Therefore, assuming that $\overline{D_{\delta+\lambda}\left(\mu_{1}\right)}=0$, we can find a $\lambda_{1}$ so that all of the conditions for a deformation are satisfied.

If $\lambda_{1}+\tau$ gives another solution, then $D_{\mu}(\tau)=0$ and $D_{\delta+\lambda}(\tau)=$ 0 . Because we do not allow elements of $C^{0,1}$ as cochains, these two equalities are equivalent to $[\bar{\tau}]$ being well defined.

If $d_{t}^{\prime}=\exp (t(\alpha+\gamma))$, then we obtain the following.

$$
\begin{aligned}
{[\delta, \alpha+\gamma] } & =0 \\
\lambda_{1}^{\prime} & =\lambda+[\lambda, \alpha+\gamma] \\
\mu_{1}^{\prime} & =\mu_{1}+[\mu, \alpha+\gamma] .
\end{aligned}
$$

Thus, up to equivalence a deformation is given by a $D_{\mu}$-cohomology class $\bar{\mu}_{1}$. If we fix $\mu_{1}$, and then look at the variation in $\tau$, one also obtains that up to equivalence, the deformation is determined by the $D_{\bar{\delta}+\bar{\lambda}}$-cohomology class $[\bar{\tau}]$ of the $D_{\mu}$-cohomology class $\bar{\tau}$. Thus we have shown

Theorem 6.2. The infinitesimal deformations of a module $M$ with Lie algebra structure $\mu$ over a Lie algebra $\delta$, allowing the algebra structure $\mu$ on $W$ and module structure $\lambda$ to vary are classified by 
(1) $D_{\mu}$ cohomology classes $\bar{\mu}_{1}$ of $D_{\mu}$-cocycles $\mu_{1}$ lying in $C^{2,0}$.

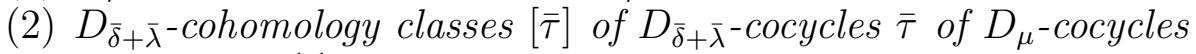
$\tau$ lying in $C^{1,1}$.

\section{Conclusions}

The authors original intent was to give a brief review of the theory of extensions of Lie algebras and then extend these notions to the $L_{\infty}$ algebra case. However, it turned out that in the course of translating the relevant ideas about extensions to the language of codifferentials, we discovered that some of the material is not readily accessible in the literature. For example, we could not locate a reference for Theorem (4.4), although it seems to be fundamental in the classification of extensions. The language of codifferentials, with its emphasis on the $\mathbb{Z}_{2}$-graded Lie algebra structure of the cochains, is natural for the description of extensions, and therefore, the results seem to us to be easier to see in this formulation.

An extension is determined by four coderivations, and the underlying theme of this paper was to study what occurs when two of them are fixed and the other two allowed to vary. In each case, we were able to give a cohomological condition which one of the two structures must satisfy in order that an extension, or deformation of an extension, existed. Then, given any one of the second type of structures yielding an extension, the variation in the second was also given by a purely cohomological condition. It was necessary to cast things in terms of the variation in the second structure because the set of second structures which give a solution in terms of the first structure form an affine space.

In studying deformations of extensions, we looked at three problems, the deformation of an extension given by varying the module and cocycle structures, and deformations given by a module structure, either by holding the algebra structure on the module fixed and varying the module structure and the algebra structure on the quotient, or by holding the algebra structure on the quotient fixed and varying the algebra structure on the module, as well as the module structure. All three of these problems could have been solved by simply pointing out that an infinitesimal deformation of an extension given by a codifferential $d$ is classified by the cohomology $H(d)$. But this approach would not give separate criteria on the structures we allow to vary, so although correct, it is not the answer we were looking for.

In [5, 6], moduli spaces of 3 and 4 dimensional Lie algebras were studied. Our basic approach to the classification of the Lie algebras 
was to construct Lie algebras of higher dimension as extensions of Lie algebras of lower dimension. Of course, this is the fundamental idea behind the classical decomposition theory of Lie algebras, so our approach is nothing new in this regard. What we discovered that was a new perspective was that by decomposing the moduli space in a careful manner, one could show that the moduli spaces have natural stratifications by orbifolds. The manner in which we constructed the moduli spaces by extensions was quite helpful in discovering that stratification.

\section{REFERENCES}

[1] Dmitry Alekseevski, Peter W. Michor, and Wolfgang Ruppert, Extensions of super Lie algebras, Journal of Lie Theory 15 (2005), no. 1, 125-134.

[2] B. Feigin and D. Fuchs, Cohomologies of Lie groups and Lie algebras, Encyclopaedia of Mathematical Sciences, vol. 21, pp. 125-215, Springer Verlag, 2000.

[3] A. Fialowski and D. Fuchs, Construction of miniversal deformations of Lie algebras, Journal of Functional Analysis (1999), no. 161(1), 76-110.

[4] A. Fialowski and M. Penkava, Deformation theory of infinity algebras, Journal of Algebra 255 (2002), no. 1, 59-88, math.RT/0101097.

[5] Lersal deformations of three dimensional Lie algebras as $L_{\infty}$ algebras, Communications in Contemporary Mathematics 7 (2005), no. 2, 145-165, math.RT/0303346.

[6] - Deformations of four dimensional lie algebras, Communication in Contemporary Mathematics (to appear), math.RT/0512354.

[7] Cartan H. and S. Eilenberg, Homological algebra, Princeton University Press, Princeton, NJ, 1956.

[8] M. Levy-Nahas and R. Seneor, First order deformations of Lie algebra representations, e(3) and Poincaré examples, Communications in Mathematical Physics 9 (1968), 242-266.

[9] S MacLane, Homology, Springer-Verlag, 1963.

[10] K. Neeb, Lie algebra extensions and higher order cocycles, Journal of Geometry and Symmetry in Physics 5 (2006), 48-74.

[11] _ Non-abelian extensions of topological Lie algebras, Communications of Algebra 34 (2006), 991-1041.

[12] A. Nijenhuis and R. Richardson, Deformation of lie algebra structures, Jour. Math. Mech. 17 (1967), 89-105.

[13] J.D. Stasheff, The intrinsic bracket on the deformation complex of an associative algebra, Journal of Pure and Applied Algebra 89 (1993), 231-235.

EÖtvÖs LorÁnd University, BudApest, Hungary

E-mail address: fialowsk@cs.elte.hu

University of Wisconsin, Eau Claire, WI 54702-4004

E-mail address: penkavmr@uwec.edu 